\title{
CDCP1 overexpression drives prostate cancer progression and can be targeted in vivo
}

\begin{abstract}
Abdullah Alajati, 1,2 Mariantonietta D’Ambrosio,, ${ }^{1,2,3}$ Martina Troiani,, ${ }^{1,2}$ Simone Mosole, ${ }^{1,2}$ Laura Pellegrini, ${ }^{1,2}$ Jingjing Chen, ${ }^{1,2,3}$ Ajinkya Revandkar, ${ }^{1,2,3}$ Marco Bolis, , Jean-Philippe Theurillat, ${ }^{1,2}$ Ilaria Guccini,, 1,2 Marco Losa,, ${ }^{1,2}$ Arianna Calcinotto, ${ }^{1,2}$ Gaston De Bernardis, ${ }^{1,2}$ Emiliano Pasquini, ${ }^{1,2}$ Rocco D’Antuono, ${ }^{4}$ Adam Sharp, ${ }^{5}$ Ines Figueiredo, ${ }^{5,6}$ Daniel Nava Rodrigues, ${ }^{5,6}$ Jonathan Welti, ${ }^{5,6}$ Veronica Gil,, ${ }^{5,6}$ Wei Yuan, ${ }^{5,6}$ Tatjana Vlajnic, ${ }^{7}$ Lukas Bubendorf, ${ }^{7}$ Giovanna Chiorino, ${ }^{8}$ Letizia Gnetti, ${ }^{9}$ Verónica Torrano, ${ }^{10,11,12}$ Arkaitz Carracedo, ${ }^{10,11,12,13}$ Laura Camplese, ${ }^{14}$ Susumu Hirabayashi, ${ }^{14}$ Elena Canato, ${ }^{15}$ Cianfranco Pasut, ${ }^{15}$ Monica Montopoli, ${ }^{15}$ Jan Hendrik Rüschoff,, ${ }^{16}$ Peter Wild, ${ }^{16}$ Holger Moch, ${ }^{16}$ Johann De Bono, ${ }^{5,6}$ and Andrea Alimonti ${ }^{1,2,3,17,18}$

IInstitute of Oncology Research (IOR), Oncology Institute of Southern Switzerland (IOSI), Bellinzona, Switzerland. 'Universita' della Svizzera Italiana, Lugano, Switzerland. ${ }^{3}$ Faculty of Biology and Medicine, University of Lausanne UNIL, Lausanne, Switzerland. ${ }^{4}$ Institute for Research in Biomedicine (IRB), Bellinzona, Switzerland. ${ }^{5}$ Division of Clinical Studies, Institute of Cancer Research, London, United Kingdom. ${ }^{6}$ Royal Marsden NHS Foundation Trust, London, United Kingdom. ${ }^{7}$ Institute for Pathology, University Hospital Basel, Basel, Switzerland. ${ }^{8}$ Fondazione Edo ed Elvo Tempia, Via Malta, Biella, Italy. ${ }^{9}$ Pathology Unit, University Hospital of Parma, Parma, Italy. ${ }^{10}$ Center for Cooperative Research in Biosciences (CIC bioGUNE), Basque Research and Technology Alliance (BRTA), Derio, Spain. "Biochemistry

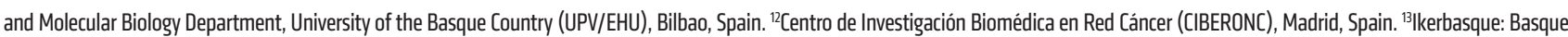
Foundation for Science, Bilbao, Spain. ${ }^{14}$ MRC London Institute of Medical Sciences (LMS), Imperial College London, London, United Kingdom. ${ }^{15}$ Department of Pharmaceutical and Pharmacological Sciences, University of Padua, Padua, Italy. ${ }^{16}$ Institute of Pathology and Molecular Pathology, University Hospital Zurich, Zurich, Switzerland. ${ }^{17}$ Department of Medicine, University of Padua, Padua, Italy. ${ }^{18}$ Department of Health Sciences and Technology, Eidgenössische Technische Hochschule Zürich (ETH), Zurich, Switzerland.
\end{abstract}

The mechanisms by which prostate cancer shifts from an indolent castration-sensitive phenotype to lethal castrationresistant prostate cancer (CRPC) are poorly understood. Identification of clinically relevant genetic alterations leading to CRPC may reveal potential vulnerabilities for cancer therapy. Here we find that CUB domain-containing protein 1 (CDCP1), a transmembrane protein that acts as a substrate for SRC family kinases (SFKs), is overexpressed in a subset of CRPC. Notably, CDCP1 cooperates with the loss of the tumor suppressor gene PTEN to promote the emergence of metastatic prostate cancer. Mechanistically, we find that androgens suppress CDCP1 expression and that androgen deprivation in combination with loss of PTEN promotes the upregulation of CDCP1 and the subsequent activation of the SRC/MAPK pathway. Moreover, we demonstrate that anti-CDCP1 immunoliposomes (anti-CDCP1 ILs) loaded with chemotherapy suppress prostate cancer growth when administered in combination with enzalutamide. Thus, our study identifies CDCP1 as a powerful driver of prostate cancer progression and uncovers different potential therapeutic strategies for the treatment of metastatic prostate tumors.

\section{Introduction}

Castration-resistant prostate cancer (CRPC) is the second leading cause of death among men in developed countries (1). Although second-generation androgen-deprivation therapies (ADTs) have been successfully used to treat CRPC, patients develop resistance and eventually succumb to the disease (2). Mechanisms of resistance in CRPCs include, among others, activation of androgen receptor (AR) (i.e., AR amplification, mutations, or splicing variants) and upregulation of signaling pathways promoting AR independent growth, such as the PI3K/AKT and MAPK pathways that are mutually deregulated in CRPCs (3-6). Although in metastatic prostate cancers the PI3K signaling pathway is activated by the loss or mutations of the tumor suppressor PTEN (7), the mecha-

Authorship note: A. Alajati and MD contributed equally to this work. Conflict of interest: A. Alimonti is a cofounder of and owns stock in OncoSense. Copyright: @ 2020 Alajati et al. This is an open access article published under the terms of the Creative Commons Attribution 4.0 International License. Submitted: June 18, 2019; Accepted: January 22, 2020; Published: April 6, 2020 Reference information: J Clin Invest. 2020;130(5):2435-2450.

https://doi.org/10.1172/JCI131133. nism by which the MAPK pathway is upregulated remains partially unknown. Interestingly, activation of the MAPK pathway by K-RAS in a PTEN-deficient prostate cancer mouse model leads to the development of metastatic prostate cancer (8). However, mutations of either KRAS or BRAF account for only a minority of human prostate cancer cases $(5,9,10)$. Thus, the identification of new regulators of this pathway, in the context of PTEN-null prostate cancers, would open the way to new effective therapies for the treatment of this disease. The Pten-null prostate conditional mouse model provides an excellent tool to study prostate tumorigenesis. Pten deficiency in the mouse prostate epithelium leads to benign prostate tumors characterized by a senescence response that opposes tumor progression $(11,12)$. Therefore, this model can be used to identify pathways or genes that serve as the "second hit" for the evasion of senescence, acquisition of metastatic potential, and the preclinical validation of new therapies in this setting (12-14).

CUB domain-containing protein 1 (CDCP1), also known as SIMA135 (15), gp140 (16), CD318 (17), or Trask (18), is a transmembrane protein that is frequently overexpressed in a variety of human cancers (19-21). Several papers demonstrate that CDCP1 is a potent oncogene that drives cancer development, invasion, 


\section{Table 1. CDCP1-positive samples in BPH, RPE, CRPC, and metastatic $P C a$ in human prostate cancers}

$\begin{array}{lcccc} & \text { BPH } & \text { RPE } & \text { CRPC } & \text { Metastasis } \\ \text { Total } & 45 & 382 & 102 & 35 \\ \text { CDCP1-positive } & 4 & 65 & 45 & 18\end{array}$

For TMA1, $n=564$.

and metastases. In cells cultured in adherent conditions, CDCP1 overexpression promotes the activation of Src-family members (SFKs), phosphorylation of protein kinase C delta (PKC- $\delta$ ), and the upregulation of the MAPK/ERK pathway (22). In contrast with these results, recent studies reported that loss of CDCP1, in cells kept in nonadherent conditions, supports tumor cells proliferation by differentially regulating SRC activity (23-25). Interestingly, CDCP1 targeting, either with monoclonal antibodies or small molecule inhibitors, has demonstrated effectiveness at inhibiting tumor growth and metastasis in vivo (26-28). Since treatments with either SRC or MAPK inhibitors have been associated with poor tolerability in the clinic (29), CDCP1 targeting could represent an excellent and alternative therapeutic option. In the present manuscript, we show that CDCP1 is overexpressed in a subset of advanced human CRPCs, and cooperates with loss of PTEN to promote the emergence of this disease. Moreover, we have found that AR represses CDCP1 transcription, whereas ADTs promote the upregulation of CDCP1 in tumor cells harboring PTEN deletions, thereby increasing the activation of the SRC/MAPK pathway. Notably, treatment of anti-CDCP1 ILs loaded with chemotherapy in combination with enzalutamide substantially inhibits prostate tumor progression. Our results introduce what we believe is a previously unknown and exciting therapeutic strategy to treat PTEN-deficient prostate cancer patients.

\section{Results}

CRPC and metastatic prostate tumors exhibit elevated expression of CDCP1, and overexpression of CDCP1 correlates with PTEN loss. To assess the clinical relevance of CDCP1 in human prostate cancer (human $\mathrm{PCa}$ ), we examined 2 different tumor microarrays (TMAs), including a total of 990 cases spanning benign, primary, and metastatic PCa (30-32). Immunohistochemical (IHC) analysis showed that while a large portion of prostate tumors analyzed did not express CDCP1, a subset (48\%) of CRPC and metastatic tumor samples expressed a high level of CDCP1 (Figure 1, A and B, Supplemental Figure 1A, and Tables 1 and 2; supplemental material available online with this article; https://doi. org/10.1172/JCI131133DS1). In line with these findings, analysis of consecutive tumor samples from a longitudinal study revealed that CDCP1 was upregulated in PCa patients during the transition from hormone-sensitive to CRPC (Supplemental Figure 1B). Intriguingly, high levels of CDCP1 correlated with decreased levels of PTEN in both primary, CRPC, and metastatic prostate tumor samples (Figure 1, C and D, and Tables 3 and 4). PTEN is one of the most frequently altered tumor suppressor genes in $\mathrm{PCa}$, where it accounts for prostate tumor initiation and progression (5). The frequency of tumors displaying a low level of
PTEN and high levels of CDCP1 increased in CRPCs and metastatic tumors when compared with primary tumors, thereby validating the clinical relevance of this anti-correlation (Figure 1, C and $\mathrm{D}$, and Tables 3 and 4). Additionally, bioinformatics analysis evaluating different data sets confirmed the existence of an anticorrelation between PTEN and CDCP1 mRNA levels (Supplemental Figure 1, C and D). Elevated levels of CDCP1 expression were also significantly associated with PTEN genetic deletions and low CDCP1 promoter methylation in different independent data sets of PCa (Figure 1E and Supplemental Figure 1, E and F). Although patients affected by prostate tumors harboring a high level of CDCP1 had a similar disease-free survival (DFS) as patients with low CDCP1, patients with tumors expressing low levels of PTEN and an increased level of CDCP1 had a significantly shorter DFS than patients in the other categories (Figure $1 \mathrm{~F}$ and Supplemental Figure 1G). Taken together, these data validate the clinical relevance of CDCP1 and suggest that CDCP1 could cooperate with the loss of PTEN to promote highly aggressive prostate cancer.

Conditional overexpression of CDCP1 in the mouse prostate and Drosophila melanogaster initiates tumorigenesis. To model CDCP1 overexpression in cancer, we generated a CDCP1 transgenic mouse model. At first, we constructed a pCAGGS vector with a transcriptional STOP sequence flanked by loxP sites upstream of CDCP1-cDNA. The resulting pCAGGS-loxP-STOP-loxP-CDCP1 vector along with $\mathrm{PGK}-\mathrm{FlpO}$ plasmid were coelectroporated into the ColA locus of modified KH2 embryonic stem cells (ref. 33 and Supplemental Figure 2A). PCR and Southern blot analysis confirmed gene integration and recombination events (Supplemental Figure 2B). Next, we crossed $C D C P 1$ with $P B-C r e 4$ mice for prostate-specific expression of CDCP1 (34). IHC, reverse transcription PCR (RT-PCR), and Western blot analyses were performed on prostate tissues of 10-week-old CDCP1 Pb-Cre mice (CDCP1 ${ }^{\text {pcLSL/+ }}$, hereafter referred to as $\left.\mathrm{CDCP} 1^{+}\right)$and confirmed the prostatespecific expression of CDCP1 (Supplemental Figure 2, C-E). Of note, the expression of CDCP1 in a panel of human prostate tumor cell lines, patient-derived prostate cancer xenografts (PDXs), and tumors collected from $\mathrm{CDCP} 1^{+}$mice did not show significant differences in CDCP1 levels (Supplemental Figure 2F), thereby demonstrating that overexpression of CDCP1 in the mouse model is similar to the CDCP1 levels in human tumors. Next, we examined tumor incidence in $C D C P 1$ mice over 24 months. CDCP1 mice developed prostate hyperplasia between 4 and 6 months of age at $50 \%$ penetrance. $C D C P 1$ mice between 7 and 9 months of age developed a high penetrance of PIN (prostatic intraepithelial neoplasia) lesions characterized by multilayered epithelial cells with features of nuclear atypia. These mice further developed high-

\section{Table 2. CDCP1 membranous staining in TMA1 tumors from BPH/ RPE and CRPC/metastasis patients}

$\begin{array}{lcc} & \text { Total } & \text { CDCP1-positive } \\ \text { BPH/RPE } & 427 & 69 \\ \text { CRPC/metastasis } & 137 & 63\end{array}$

The $\chi^{2}$ test was used for statistical analysis. $\chi^{2}=29.9301 . P<0.00001$. The result is significant at $P<0.05$ 
A
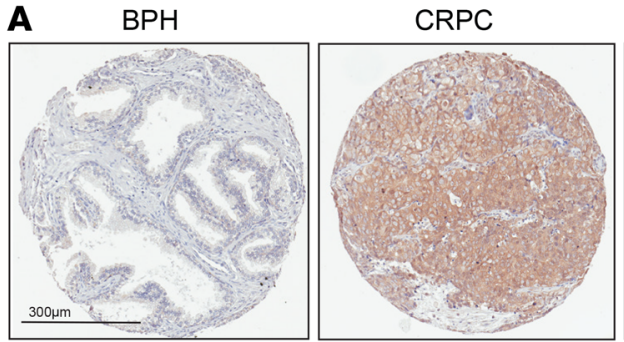

C

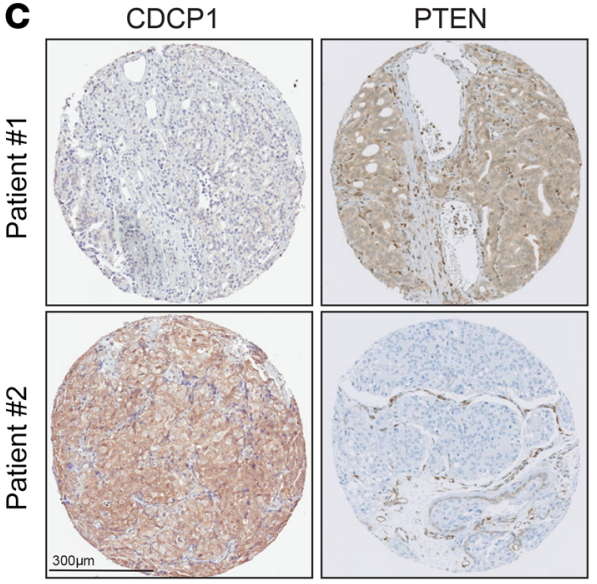

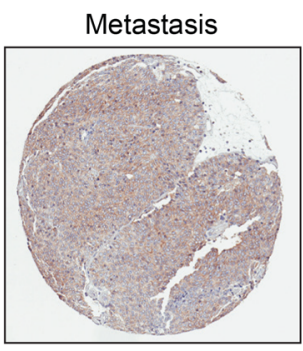

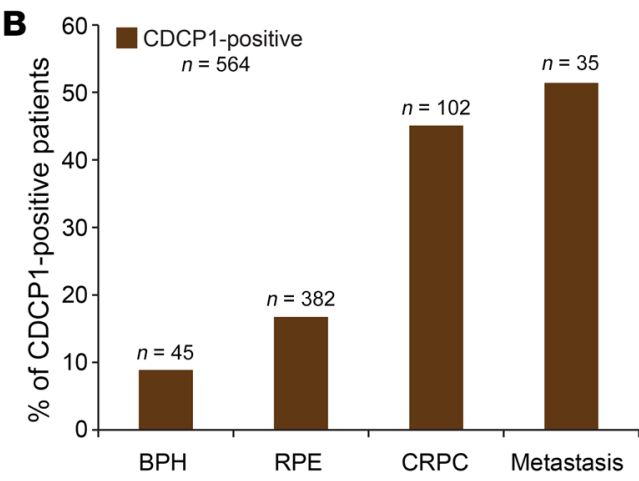

E

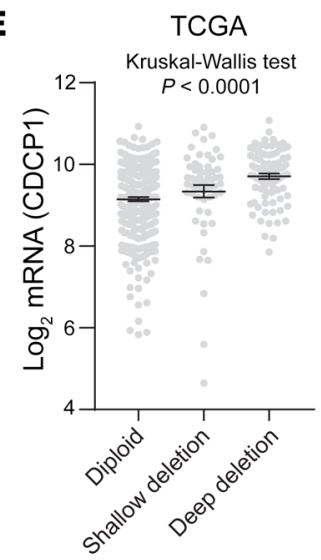

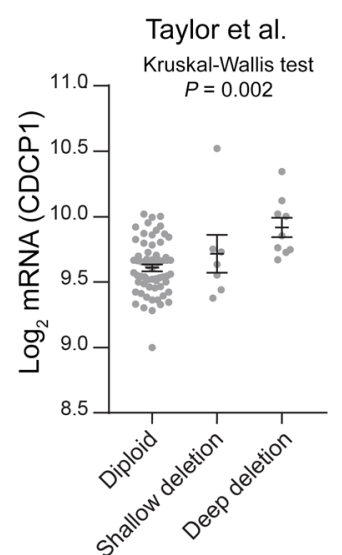

$\mathbf{F}$

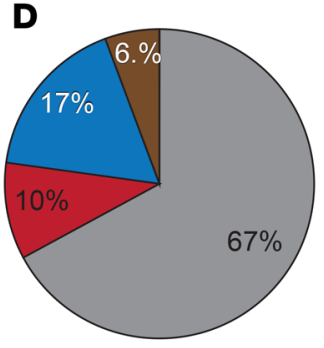

Total $n=386$

Primary tumors

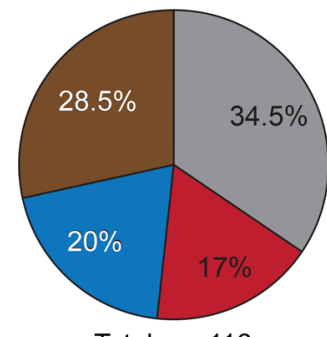

Total $n=116$

CRPC/Metastasis

$\square$ PTEN-high/CDCP1-negative

PTEN-low/CDCP1-negative

PTEN-high/CDCP1-positive

PTEN-low/CDCP1-positive

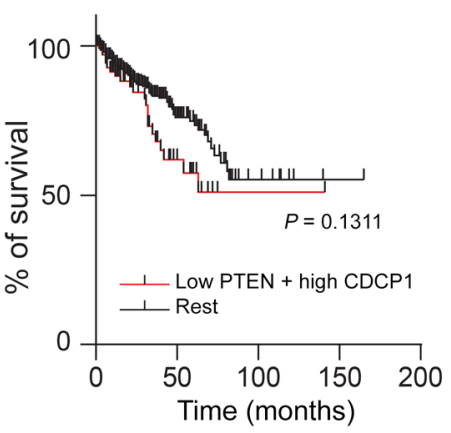

Taylor et al.

NM_022842

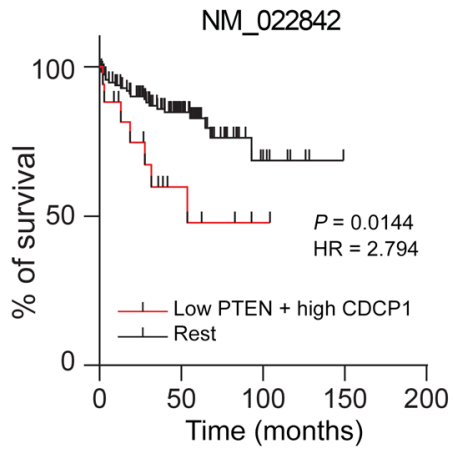

Figure 1. Advanced and metastatic prostate tumors exhibit elevated expression of CDCP1 and overexpression of CDCP1 correlate with PTEN loss. (A) Representative images of IHC staining of CDCP1 in benign prostate hyperplasia (BPH), CRPC, and distant metastasis of PCa in human prostate cancer TMA1. Scale bar: $300 \mu \mathrm{m}$. (B) Percentage of CDCP1-positive samples in BPH, preradical prostatectomy (RPE), CRPC, and metastatic PCa in human prostate cancer TMA1 $(n=564)$. (C) Representative images of IHC staining of CDCP1 and PTEN in 2 different PCa patients. Scale bar: $300 \mu \mathrm{m}$. (D) Pie graph showing the percentage of PTEN-high/CDCP1-, PTEN-high/CDCP1-, PTEN-high/CDCP1+, PTEN-low/CDCP1- and PTEN-low/CDCP1+ in primary tumors and CRPC/ metastasis. (E) Association of PTEN genomic loss to CDCP1 gene expression in TCCA (left panel) and Taylor data set (right panel) (5). Error bars indicate SEM; statistical test: Kruskal-Wallis. (F) Association of PTEN and CDCP1 expression levels with disease-free survival in the indicated patient data sets. In the Taylor data set, low PTEN indicates patients with expression signal lower than 8.74, and high CDCP1 indicates patients with expression signal higher than 11.19. In TCCA, low PTEN indicates patients with expression signal lower than 10.19, and high CDCP1 indicates patients with expression signal higher than 9.49. HR, hazard ratio. Statistical test: Mantel-Cox.

grade PIN (HGPIN) lesions after 14 months of age with $100 \%$ penetrance and showed high Ki67 expression (Figure 2, A-D, and Supplemental Figure 2G). In parallel, Western blot analysis revealed a significant increase of Src and Erk1/2 phosphorylation in the prostatic epithelium of $C D C P 1$ mice and $C D C P 1^{+}$mouse embryonic fibroblast (MEFs) derived from this model (Figure 2, $\mathrm{E}$ and F). To further validate these findings in a different model, we overexpressed both WT human CDCP1 (CDCP1-WT) and an inactive form of CDCP1 (CDCP1-delta) lacking Src-phosphorylation sites $(35,36)$ in Drosophila melanogaster. The Drosophila larval imaginal discs are a monolayer epithelium that is considered morphologically comparable to mammalian epithelia and therefore constitutes an ideal system in which to model cancer progression in vivo (37). Increased EGFR/Ras signaling has been previously shown to promote the formation of bristles located on the dorsal part of the fly thorax (notum) (also referred to as macrochaetae formation), 
Table 3. PTEN and CDCP1 membranous staining in primary TMA1 tumors from PCa patients

$\begin{array}{lcc} & \text { CDCP1-negative } & \text { CDCP1-positive } \\ \text { PTEN-normal } & 259 & 39 \\ \text { PTEN-low } & 66 & 22\end{array}$

To sheer cross-linked DNA to an average fragment, the $\chi^{2}$ statistical test was used. $\chi^{2}=7.246 . P<0.007106$. The result is significant at $P<0.05$.

a tumor-like phenotype $(35,36)$. We found that overexpression of CDCP1-WT, but not CDCP1-delta, promoted extra macrochaetae formation. Note that both CDCP1 isoforms localized at the cell membrane of the salivary gland of the fly, as assessed by costaining of E-cadherin, and presented similar expression levels (Supplemental Figure 3, A-C, arrows). Interestingly, loss of 1 allele (50\% reduction) of $\operatorname{src} 42 A$ and $\operatorname{src} 64 B(35,38,39)$, the 2 Src homologs in Drosophila, suppressed extra macrochaetae formation driven by the overexpression of CDCP1-WT, demonstrating that this phenotype is Src-dependent (Supplemental Figure 3D). Collectively, this cross-species genetic approach demonstrates that CDCP1 overexpression in vivo initiates tumorigenesis.

CDCP1 cooperates with Pten loss to drive prostate cancer progression and metastasis. To further model the interplay existing between PTEN and CDCP1 in vivo, we crossed CDCP1 mice with Pten-null prostate conditional mice (Pten ${ }^{p c /-}$ ) to obtain CDCP1 Pten ${ }^{p c /-}$ double mutant mice. Although monoallelic loss or mutations in PTEN is associated with benign prostate tumors $(34,40)$, complete loss of PTEN is frequently observed in human metastatic prostate cancer (5). However, complete loss of Pten in the mouse is not sufficient to promote metastatic prostate cancer and additional genetic hits are needed to promote the onset of metastases (12). Strikingly, by the age of 25 weeks, CDCP1 Pten pc-/- $^{\mathrm{c}}$ mice developed focally invasive adenocarcinoma, which progressed to highly aggressive carcinoma at later time points, a phenotype that is never observed in $\mathrm{Pten}^{\mathrm{pc}-/-}$ mice (Figure 3A). Notably, the macroscopic analysis showed a significant increase in weight and volume of CDCP1 Pten ${ }^{\mathrm{pc}-/}$ tumor compared with its counterparts (Figure 3B). Importantly, histopathological analysis of CDCP1 Pten ${ }^{\mathrm{pc}-/-}$ mice revealed metastatic spread of epithelial tumor nodules, positive for Pan-Cytokeratin (PanK), CDCP1, and AR, to draining lumbar lymph nodes in $50 \%$ ( $n=4 / 8)$ and to the lung in $11 \%(n=1 / 9)$ of the cases analyzed (Figure $3 \mathrm{C}$ and Supplemental Figure 4, A and B). The histological features of these metastases resembled those of the primary prostate tumors (Supplemental Figure 4A). By contrast, Pten $^{\mathrm{pc}-/-}$ mice did not develop metastasis, as previously reported (12-14). Moreover, MEFs derived from CDCP1 Pten ${ }^{-/}$mice showed an increased proliferative and migratory capacity when compared with $\mathrm{Pten}^{-/-}$cells (Supplemental Figure 4, C and D). Additionally, Kaplan-Meier cumulative survival analysis showed that CDCP1 Pten ${ }^{\mathrm{pc}-/-}$ mice died or had to be euthanized due to extensive tumor burden at the age of 60 to 80 weeks (Figure 3D). Of note, none of the age-matched $\mathrm{Pten}^{\mathrm{pc}-/}$ mice died, indicating a profound effect of CDCP1 overexpression on the survival of $\mathrm{Pten}^{\mathrm{pc}-/}$ mice. Moreover, the percentage of Ki-67 positive cells was significantly higher in CDCP1 Pten ${ }^{\mathrm{pc}-1}$ mice when compared with their counterpart mice (Figure $3 \mathrm{E}$ ). At the molecular level, Western blot analysis revealed that CDCP1 Pten $^{\mathrm{pc}-/-}$ tumors showed elevated levels of Src and p-Erk1/2, whereas p-Akt was not changed compared with $\mathrm{Pten}^{\mathrm{pc}-/-}$ tumors (Figure $3 F)$. Since activated Src is known to regulate c-Myc levels (41-43), we reasoned that CDCP1 overexpression could drive c-Myc overexpression through Src. Indeed, CDCP1-overexpressing tumors showed increased levels of c-Myc expression (Figure 3F). Furthermore, IHC analysis revealed high levels of c-Myc and pErk1/2 in CDCP1 Pten ${ }^{\mathrm{pc}-/-}$ tumors compared with Pten ${ }^{\mathrm{pc}-/-}$ tumors (Figure $3 \mathrm{G}$ ).

We next checked whether CDCP1 could also promote resistance to androgen deprivation therapy (ADT) in the same setting. To this end, we performed surgical castration in both Pten ${ }^{\mathrm{pc}-/-}$ and CDCP1 Pten ${ }^{\mathrm{pc}-/-}$ mice. Although Pten $\mathrm{pc}^{\mathrm{p}-/-}$ tumors responded to castration as previously reported (44), CDCP1 Pten $\mathrm{Pc}^{\mathrm{pc}-\mathrm{-}}$ did not, as shown by tumor weight, volume, histopathological analysis, and IHC for Ki-67 (Supplemental Figure 5, A-D). Resistance to castration in CDCP1 Pten tc- $^{p-}$ tumors was associated with higher levels of p-Src, p-Erk1/2, and c-Myc when compared with Pten ${ }^{p c-/-}$ tumors, thus explaining the emergence of CRPC in this genetic background (Supplemental Figure 5, E-G). These data were additionally validated in vivo by overexpressing CDCP1 in TRAMP-C1 mouse prostate epithelial cells injected into C57BL/6 mice (TRAMP-C1CDCP1). Overexpression of CDCP1 in TRAMP-C1 cells significantly increased the levels of p-Src and p-Erk (Supplemental Figure $5 \mathrm{H}$ ), accelerated the emergence of castration-resistant prostate cancer, and shortened the survival of TRAMP-C1-CDCP1 mice when compared with the control group (Supplemental Figure 5I).

Overexpression of CDCP1 bypasses the SMAD4 senescence barrier through activation of the Src/MAPK/Myc axis. Previous evidence demonstrated that Pten ${ }^{\mathrm{pc}-/}$ mice develop indolent tumors characterized by a senescence response that acts as an intrinsic barrier to constrain prostate cancer progression $(11,12)$. Since CDCP1 accelerates tumor progression in $\mathrm{Pten}^{\mathrm{pc}-1-}$ mice, we tested whether CDCP1 overexpression in this genetic background could promote senescence evasion both in vitro and in vivo, leading to metastasis. Prostate sections of the various genotypes (WT, CDCP1, Pten ${ }^{p c-/}$, and CDCP1 $\left.P_{t e n}{ }^{p c-/}\right)$ were analyzed for senescence response by performing SA- $\beta$-gal and p-HP1 $\gamma$ staining, 2 markers of senescence in vivo (45). Although $\mathrm{Pten}^{\mathrm{pc}-/}$ tumors exhibit a strong cellular senescence response, CDCP1 Pten ${ }^{p c-/}$ tumors stained negative for both SA- $\beta$-gal and $\mathrm{p}-\mathrm{HP} 1 \gamma$ and positive for Cyclin D1, a marker of cell proliferation, thereby demonstrating that $\mathrm{CDCP} 1$ bypasses the senescence response driven by Pten loss (Figure 4A). CDCP1 Pten ${ }^{-/} \mathrm{MEFs}$ also stained negative for SA- $\beta$-gal and exhibited increased cell proliferation with an elongated phenotype when compared with $\mathrm{Pten}^{-/-} \mathrm{MEFs}$ (Supplemental Figure 6A).

\section{Table 4. PTEN and CDCP1 membranous staining in TMA1 tumors from CRPC/metastasis PCa patients}

$\begin{array}{lcc} & \text { CDCP1-negative } & \text { CDCP1-positive } \\ \text { PTEN-normal } & 40 & 20 \\ \text { PTEN-negative } & 23 & 33\end{array}$

The $\chi^{2}$ test was used for statistical analysis. $\chi^{2}=7.6471 . P<0.005686$. The result is significant at $P<0.05$. 
A
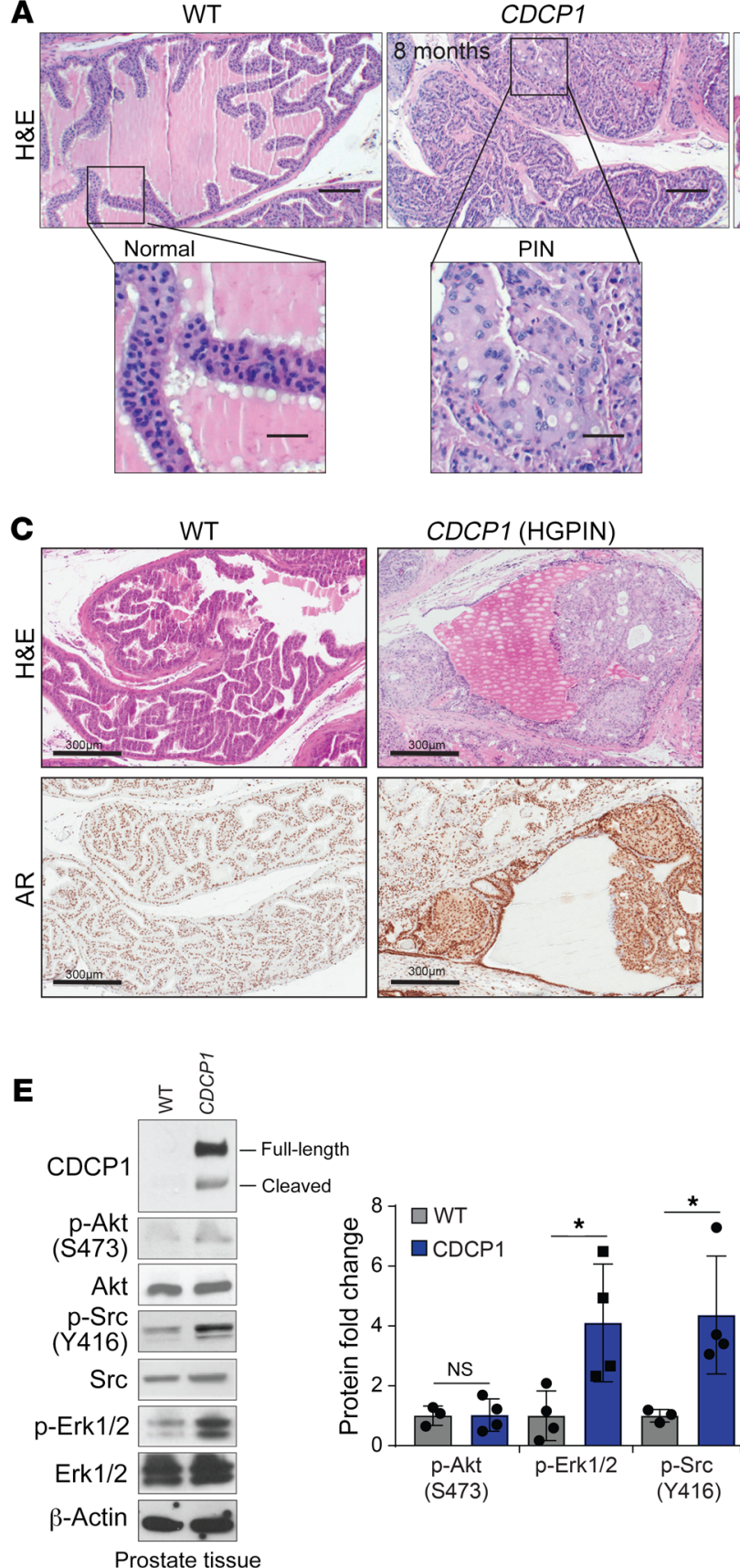

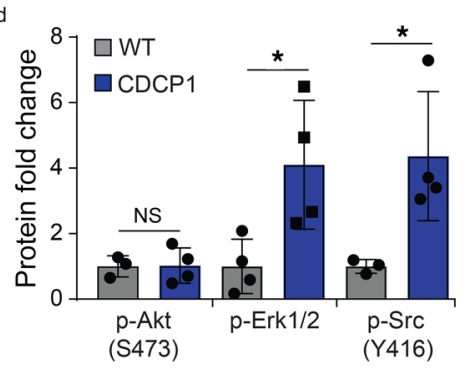

Prostate tissue
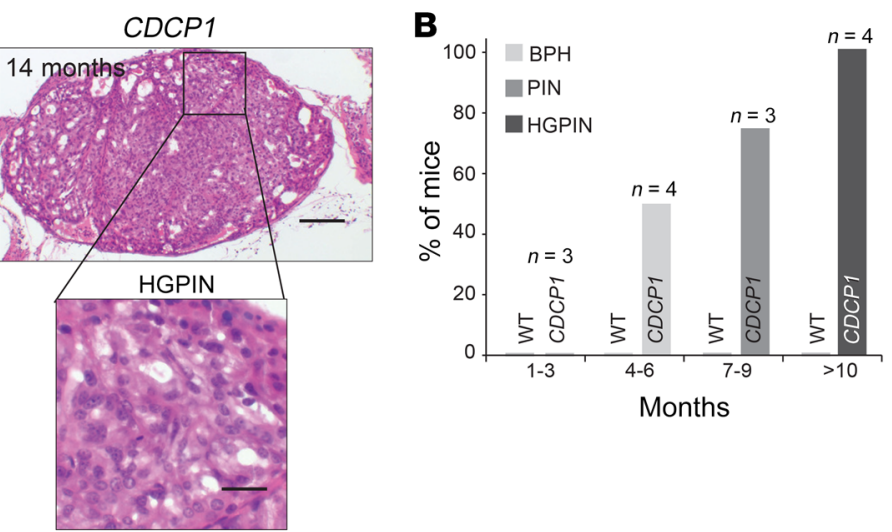

Months
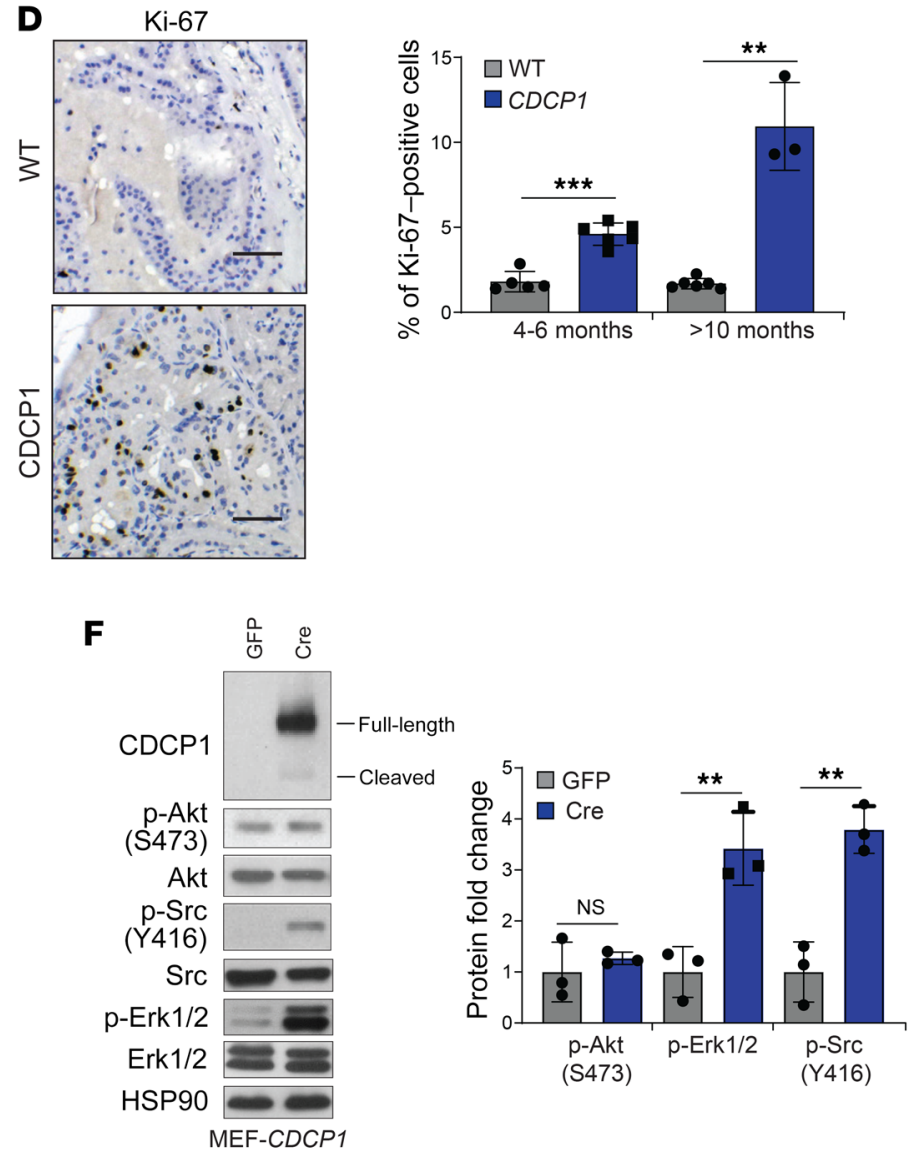

Figure 2. Conditional overexpression of CDCP1 initiates tumorigenesis. (A) Representative images of H\&E staining of anterior prostate of WT and CDCP1 mice. Scale bars: $500 \mu \mathrm{m}$. Boxes represent regions in higher magnification in WT mice, prostatic intraepithelial neoplasia (PIN), and high-grade PIN (HGPIN) in CDCP1 mice. Scale bars: $125 \mu \mathrm{m}$. (B) Histopathological characterization and quantification of the prostate in WT and CDCP1 mice. (C) IHC staining of H\&E and AR in representative anterior prostate gland of WT and CDCP1 mice affected by HCPIN. Scale bars: $300 \mu \mathrm{m}$. (D) IHC staining of Ki-67 in representative anterior prostate of WT and CDCP1 mice older than 10 months. Scale bars: $250 \mu \mathrm{m}$. Quantification of Ki-67 staining in anterior prostate of WT and CDCP1 mice at the indicated ages ( $n=3-7$ for each genotype). (E) Western blot analysis of major downstream targets of CDCP1 signaling in anterior prostates of 4-month-old WT and CDCP1 mice. Bar graph represents the fold change of normalized p-Akt, $p$-Erk1/2, and p-Src to their total proteins in CDCP1 prostates compared with WT prostates $(n=4)$. (F) Western blot analysis of major downstream targets of CDCP1 signaling in mouse embryonic fibroblasts (MEFs) from CDCP1 transgenic mice infected with retroviral vector overexpressing GFP or Cre. Bar graph represents the fold change of normalized p-Akt, $\mathrm{p}$-Erk1/2, and p-Src to their total proteins in transgenic MEF-CDCP1 mice infected with GFP or Cre retro-virus vectors $(n=3)$. Error bars indicate SD. ${ }^{*} P<0.05 ;{ }^{* *} P<0.01 ;{ }^{* *} P<0.001$. Statistical test: 2 -tailed $t$ test.

Two recent independent reports showed that TGFB/Smad4 pathway upregulation triggered by PTEN loss constrains prostate cancer progression by blocking Cyclin D1 transcription (13, 14). Of interest, overexpression of COUP-TFII, which inhibits
Smad4-dependent transcription, promotes senescence evasion by releasing Cyclin D1 expression in Pten-null cells $(13,14)$. Thus, we compared the status of several components involved in these pathways such as p53, p21, Smad4, Cyclin D1, and COUP-TFII in 

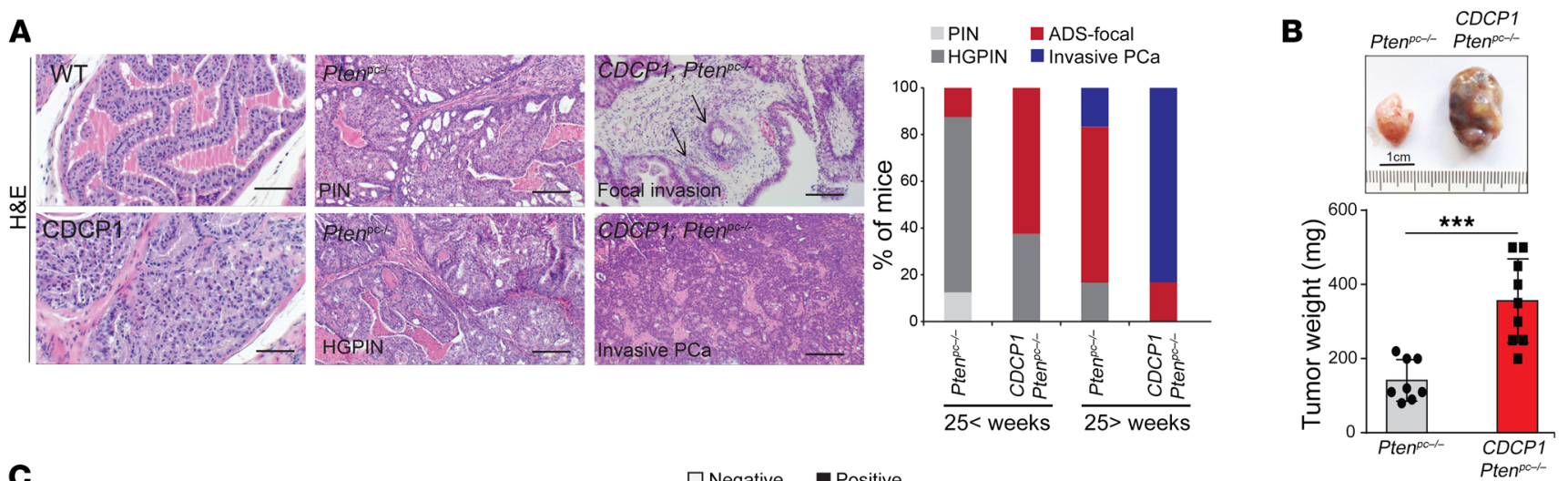

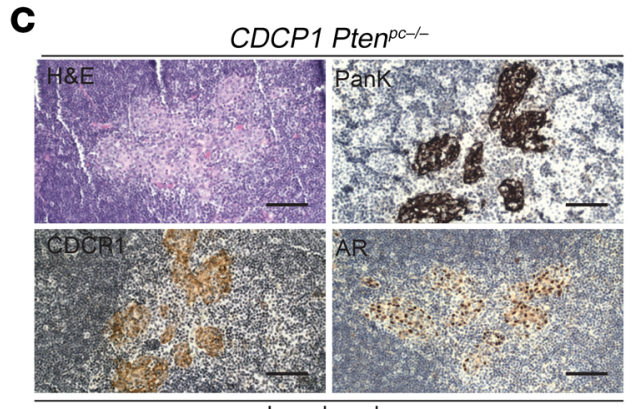

Lymph node

\section{E}

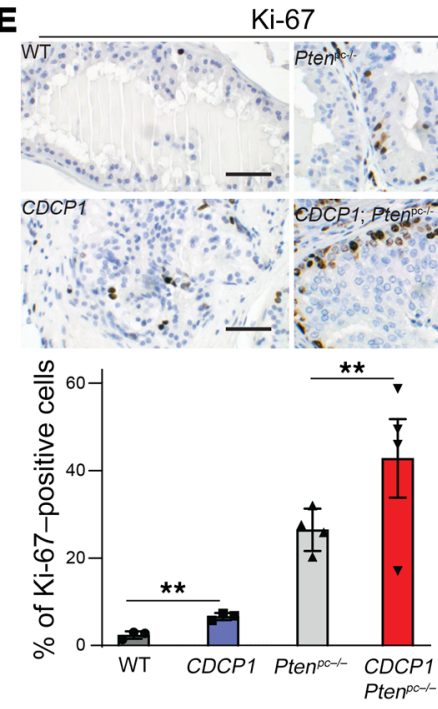

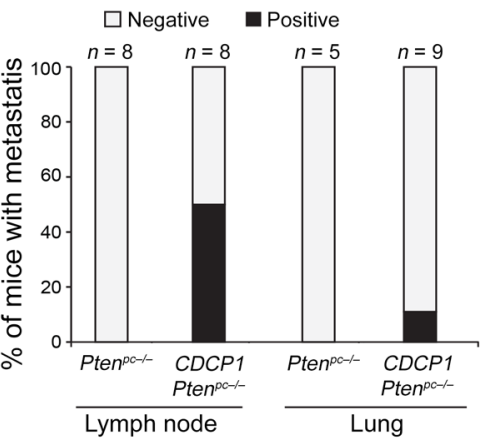
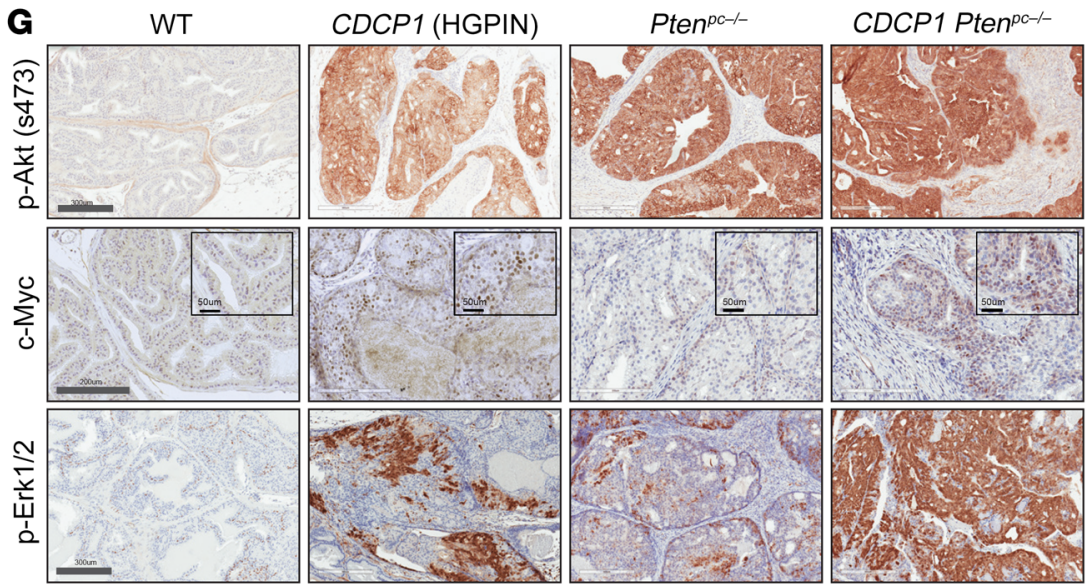

D

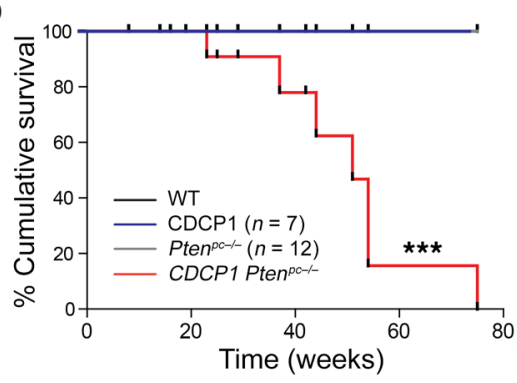

F

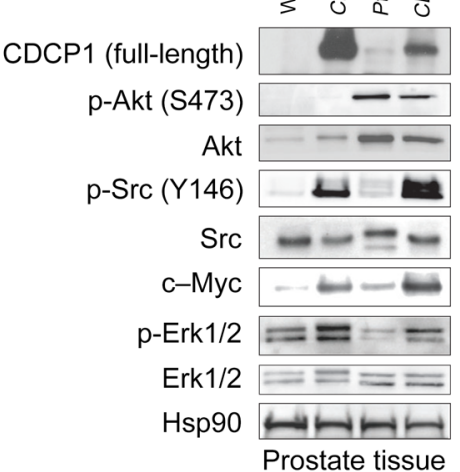

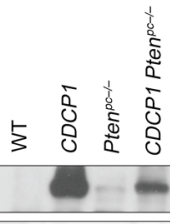

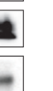
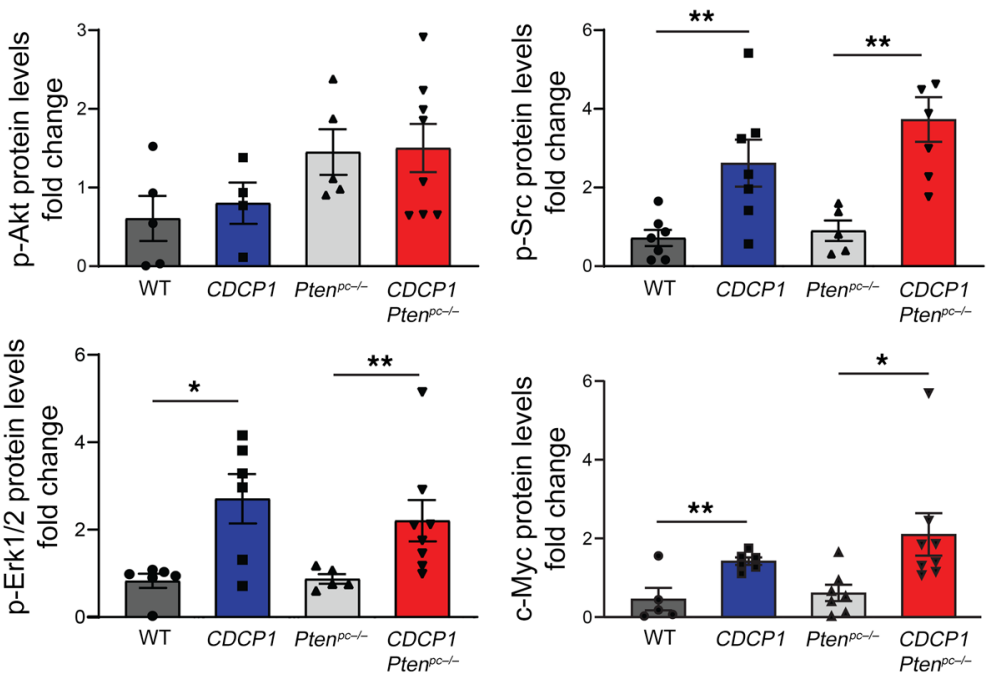
Figure 3. CDCP1 cooperates with Pten loss to drive prostate cancer progression and metastasis. (A) Representative images of H\&E staining of anterior prostate of WT, CDCP1, Pten ${ }^{p c-/-}$, and CDCP1 Pten $n^{p c-/-}$ mice at the age of 10 months. Scale bars: $500 \mu \mathrm{m}$. Bar graph representing the percentage of mice with PIN, HGPIN, ADS-focal, and invasive PCa. (B) Bar graph representing tumor weight of $P t e n^{p c-/-}$ and $C D C P 1$ ten $^{p c-/-}$ mice, insets represent anterior prostate of Pten ${ }^{p c-1-}$ and CDCP1 Pten ${ }^{p c-/}$. Scale bar: $1 \mathrm{~cm}$. (C) Representatives images of H\&E, Pan-cytokeratin (PanK), CDCP1, and AR staining of lumbar lymph node metastases in CDCP1 Pten ${ }^{p c-/-}$ mice at 10 months of age $(n=4 / 8)$. Scale bars: $250 \mu \mathrm{m}$. Graph shows the percentage of mice with lymph node and lung metastasis. (D) Cumulative survival of WT, CDCP1, Pten ${ }^{p c-/-}$, and CDCP1 Pten ${ }^{p c-/-}$ mice. (E) Representative images of Ki-67 staining in anterior prostate of WT, CDCP1, $P$ ten $^{p c-/}$, and CDCP1 $P$ ten $^{p c-/-}$ mice (3 months old). Scale bars: $125 \mu \mathrm{m}$. Quantification of Ki-67 staining in anterior prostate of indicated genotypes $(n=3-4$ for each genotype). (F) Western blot analysis and protein fold change quantification of specified proteins in anterior prostate glands from the indicated genotypes at 20 weeks of age. Graphs show protein fold change quantification of p-Src, p-Erk1/2, p-Akt, and c-Myc $(n=5-7)$. (C) Immunohistochemistry staining of p-AKT, p-ERK1/2, and c-Myc of anterior prostates of WT, CDCP1, Pten ${ }^{p c-/-}$, and CDCP1 Pten ${ }^{p c-/-}$ mice. Scale bars: $300 \mu \mathrm{m}$ (p-AKT, p-ERK1/2); $200 \mu \mathrm{m}$ (c-Myc); $50 \mu \mathrm{m}$ (inset). Error bars indicate SD for $\mathbf{B}$ and $\mathbf{E}$ and SEM for $\mathbf{F} .{ }^{*} P<0.05 ;{ }^{* *} P<0.01$; ${ }^{* *} P<0.001$. The following statistical tests were used: unpaired 2-tailed $t$ test for $\mathbf{B}$ and $\mathbf{E}$, log-rank (Mantel-Cox) test for $\mathbf{D}$, and 1-tailed $t$ test for $\mathbf{F}$.

Pten $^{\mathrm{pc}-/-}$ and CDCP1 Pten ${ }^{\mathrm{pc}-/-}$ tumor samples. Although our analysis showed that Smad4 and p53 expression did not change in CDCP1 Pten-null MEFs and tumors compared with control groups, Cyclin D1 and COUP-TFII levels were significantly altered (Figure 4, B and C, and Supplemental Figure 6, B and C). These data suggest that CDCP1 allows Pten-null benign tumors to acquire metastatic potential through the evasion of the TGFB-induced senescence barrier by increasing the level of COUP-TFII. We next tried to understand the mechanism by which CDCP1 controlled COUPTFII levels. Interestingly, COUP-TFII, c-Myc, and Cyclin D1 mRNA and protein levels were significantly reduced in CDCP1 $\mathrm{Pten}^{-/-}$MEFs upon treatment with saracatinib, a selective inhibitor of Src (ref. 46, Figure 4D, and Supplemental Figure 6D). Of note, the saracatinib treatment led to a profound arrest in the proliferation and reactivation of senescence in CDCP1 $\mathrm{Pten}^{-/-} \mathrm{MEFs}$ (Figure $4 \mathrm{E}$ and Supplemental Figure 6E). Since Src controls the levels of c-Myc, we next checked whether c-Myc could regulate COUP-TFII levels. We found that c-Myc inactivation in CDCP1 Pten ${ }^{-/-}$MEFs phenocopied the results obtained with the Src inhibitor (Figure 4, F and G, and Supplemental Figure 6, F and G). In line with this evidence, the analysis of the COUP-TFII promoter revealed the presence of multiple MYC-binding sites (Supplemental Figure $6 \mathrm{H})$. Chromatin immunoprecipitation (ChIP) assays confirmed that c-Myc specifically binds to the promoters of COUP-TFII in CDCP1 Pten ${ }^{-/-}$but not to those in Pten ${ }^{-/}$MEFs. Additional ChIP analysis showed increased binding of c-Myc on Cyclin D1 promoter and reduced Smad4 binding affinity to the promoter of Cyclin D1 in CDCP1 Pten ${ }^{-/-}$MEFs compared with Pten ${ }^{-/-}$(Figure 4H). Altogether, these data demonstrate that in CDCP1 $\mathrm{Pten}^{\mathrm{pc}-/-}$ tumors, increased levels of c-Myc promote activation of COUP-TFII, which prevents Smad 4 from binding to the promoter of Cyclin D1.

To further assess the relevance of these findings in human prostate cancer cells, we checked whether inhibition of CDCP1 could drive senescence activation in prostate cancer harboring elevated levels of CDCP1. We therefore depleted CDCP1 in PC3, a PTEN TP53-deficient human prostate cancer cell line, by using 2 independent sh-RNAs (Supplemental Figure 7A). Remarkably, the silencing of CDCP1 inhibited the 3D proliferation of PC3 cells (Supplemental Figure 7B) and promoted senescence (Supplemental Figure 7, C and D). These results were also validated in vivo by injecting PC3 sh-CDCP1 and control cells in SCID mice (Supplemental Figure 7, E and F). Of note, CDCP1-depleted PC3 tumors showed a significant decrease in c-MYC, COUP-TFII, and Cyclin D1 levels in parallel with the reduction of SRC phosphorylation (Supplemental Figure 7G). Together, these data demonstrate that CDCP1 inhibition promotes senescence by suppressing c-MYC levels in human prostate cancer cells. Downregulation of CDCP1 in LNCaP-abl cells that present an increased level of CDCP1 compared with LNCaP parental cells decreased proliferation and increased senescence (Supplemental Figure 7, H-J).

Androgen deprivation induces CDCP1 expression in PTENdeficient cells. Since PTEN-deficient CRPC tumors display high CDCP1 levels, and PTEN can regulate the levels and transcriptional activity of AR (47), we formed the hypothesis that AR could control the levels of CDCP1. Bioinformatics analysis in CRPC cases revealed that $\mathrm{AR}$ expression and $\mathrm{AR}$ activity inversely correlated with CDCP1 expression in prostate tumors (Supplemental Figure $8, \mathrm{~A}$ and $\mathrm{B}$ ). To further validate these data in vitro, we cultured the androgen-sensitive PTEN-null LNCaP cell line in full androgen deprivation (FAD) condition (absence of androgens and presence of enzalutamide) for more than 40 days and waited until these cells developed resistance (Figure 5A). CDCP1 levels increased in cells resistant to enzalutamide (androgen deprivation insensitive, ADI) when compared with enzalutamide-sensitive cells (androgen deprivation sensitive, ADS). This upregulation was associated with the concomitant activation of p-SRC, p-ERK1/2, and c-MYC and to evasion of senescence driven by enzalutamide treatment (ref. 48, Figure 5B, and Supplemental Figure 8C). These results prompted us to investigate whether AR could regulate the mRNA expression of CDCP1. Although FAD treatment enhanced CDCP1 levels, dihydrotestosterone (DHT) stimulation reduced its expression at both mRNA and protein levels in LNCaP ADS cells (Figure $5 C)$. In addition, overexpression of AR reduced the mRNA and protein levels of CDCP1 in the $\mathrm{AR}^{-}$prostate cancer cell line PC3 (Figure 5D and Supplemental Figure 8D). In contrast, overexpression of a mutated form of AR that lacked the DNA binding domain in PC3 failed to promote the downregulation of CDCP1 (Figure 5E). ChIP-quantitative PCR (ChIP-qPCR) analysis in LNCAP cells, showed that the AR could bind to the CDCP1 proximal promoter, where it inhibited CDCP1 transcription (Figure 5F).

We next investigated whether loss of PTEN was needed for the upregulation of CDCP1 in cells kept in FAD. Indeed, CDCP1 levels increased in PTEN-null LNCaP cells but not in the PTEN-WT LAPC4 and VCaP cell lines kept in FAD (Figure 5G). In line with these findings, we found that in the ADT-insensitive cell lines PC3 and 22RV1, FAD did not upregulate CDCP1 levels (Supplemental Figure 8E). Interestingly, inhibition of PI3K in LNCaP cells, but not in 22RV1 cells, promoted a downregulation of CDCP1 in cells kept in FAD (Figure $5 \mathrm{H}$ and Supplemental Figure 8F). This was associated with the concomitant upregulation of AR levels in the same cells. These data are in agreement with previous findings 
A

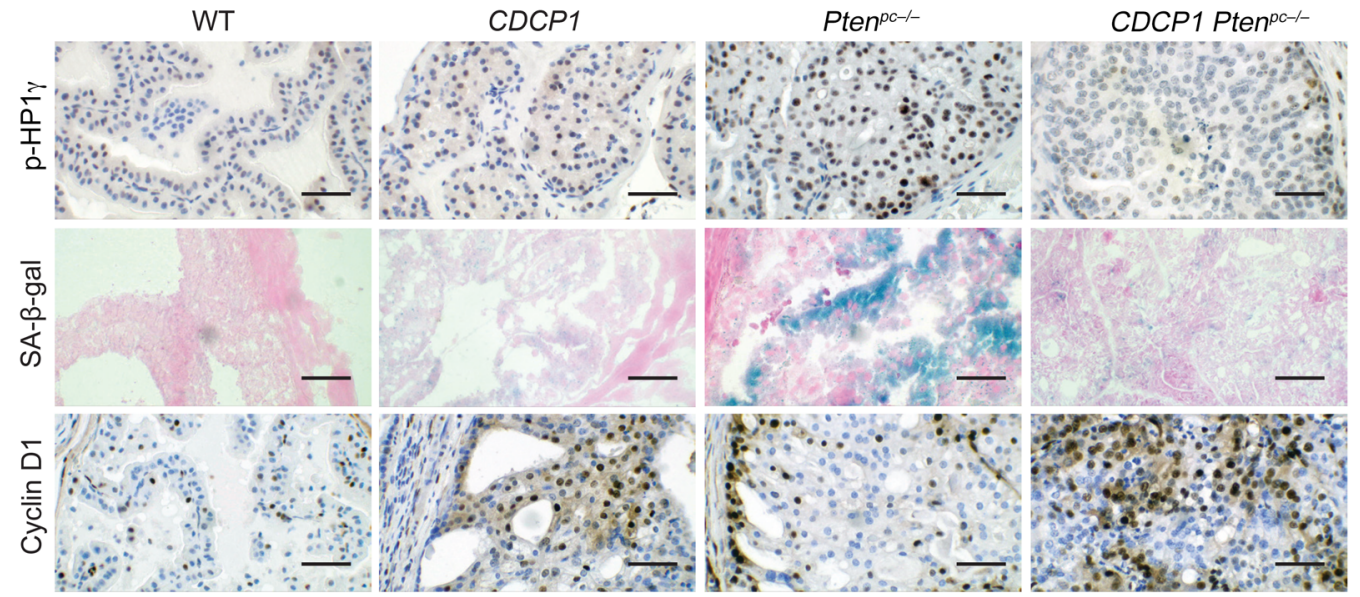

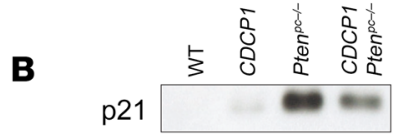

Cyclin D1

COUP-TFII

Smad4

p53

Hsp90

$$
\text { Prostate tissue }
$$
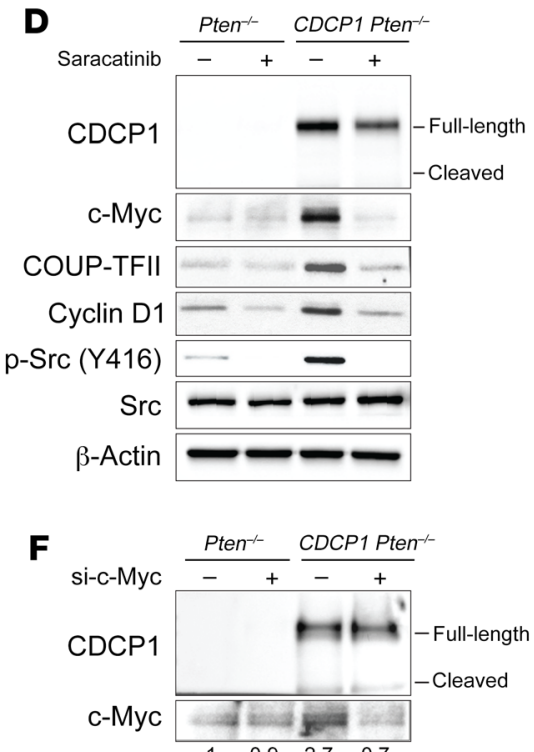

$\mathrm{p}-\mathrm{Src}(\mathrm{Y} 416)$

$$
\text { Src }
$$

$\beta$-Actin

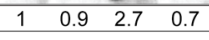

H

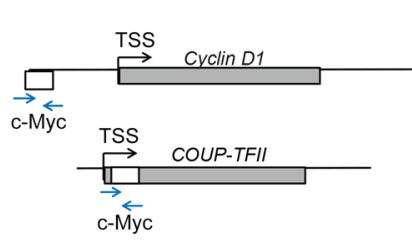

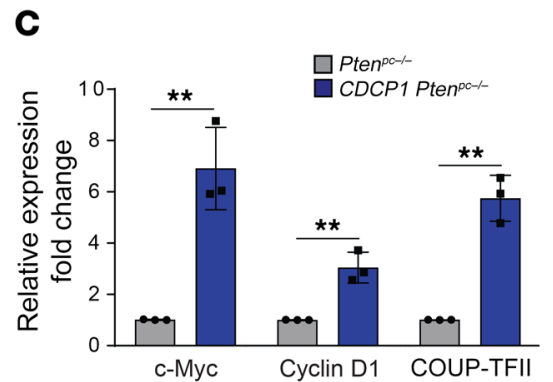

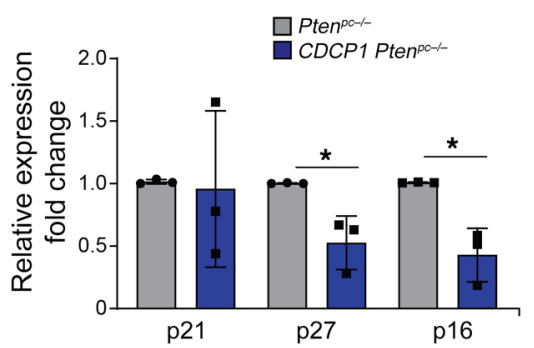

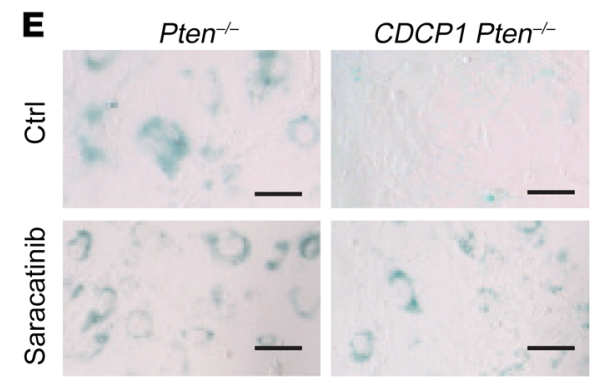
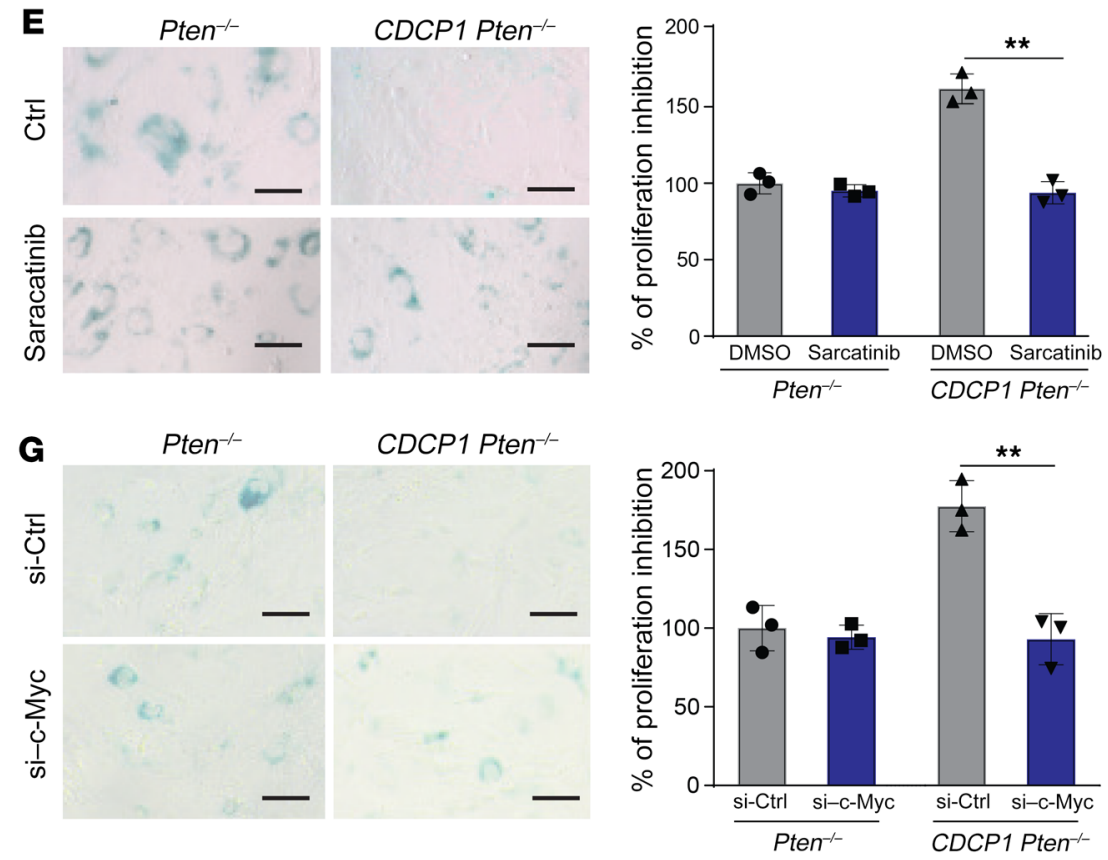

COUP-TFII

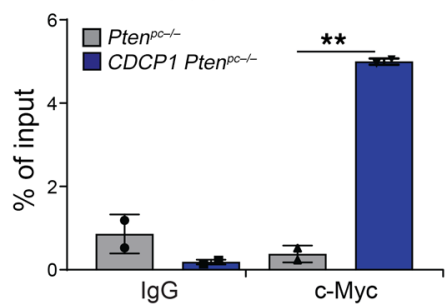

Cyclin D1

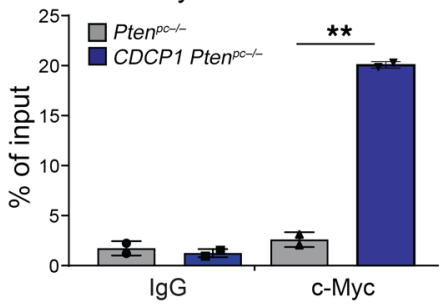

Cyclin D1

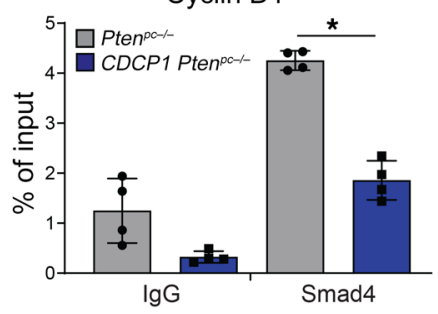


Figure 4. Overexpression of CDCP1 overcomes Pten loss-induced cellular senescence bypassing the SMAD4 barrier through activation of the Src/ MAPK/c-Myc axis. (A) Representative images of p-HP1 $\gamma$. Senescence associated- $\beta$-galactosidase (SA- $\beta$-Gal) and Cyclin D1 staining in the anterior prostates of WT, CDCP1, Pten ${ }^{p c-1-}$, and CDCP1 Pten ${ }^{p c-/-}$ mice. Scale bars: 125 $\mu \mathrm{m}$. (B) Western blot analysis of p21, Cyclin D1, COUP-TFII, Smad4, and p53 in anterior prostate glands from the indicated genotypes. (C) qRT-PCR analysis of c-Myc, Cyclin D1, COUP-TFII, p21, p27, and p16 expression in prostates from 12- to 16-week-old Pten ${ }^{p c-/-}$ and CDCP1 Pten ${ }^{p c-/-}$ mice $(n=3)$. (D) Western blot analysis of Pten $^{-/-}$and CDCP1 Pten ${ }^{-/-}$MEFs treated with saracatinib $(100 \mathrm{nM})$ for 12 hours. (E) Representative images of SA- $\beta$-Gal staining in $P_{t e^{-/-}}$and CDCP1 Pten ${ }^{-/-}$MEFs treated with saracatinib (100 nM) and DMSO for 12 hours. Scale bars: $125 \mu \mathrm{m}$. Bar graph shows the fold change in growth by crystal violet in Pten $^{-/}$and CDCP1 Pten ${ }^{-/-}$MEFs treated with saracatinib $(100 \mathrm{nM})$ or DMSO as control $(n=3)$. (F) Western blot analysis of Pten $^{-/}$ and CDCP1 Pten ${ }^{-1-}$ MEFs transfected with si-c-Myc and control si-scramble (si-Ctrl) after 48 hours. (G) Representative images of SA- $\beta$-Gal staining in Pten $^{-/-}$and CDCP1 Pten ${ }^{-/-}$MEFs transfected with si-c-Myc and si-Ctrl after 48 hours. Scale bars: $125 \mu \mathrm{m}$. Bar graph shows the fold change in growth by crystal violet in Pten $^{-/-}$and CDCP1 Pten ${ }^{-/-}$MEFs transfected with si-c-Myc and si-Ctrl $(n=3)$. (H) Schemes of Cyclin D1 and COUP-TFII promoters. qRTPCR of ChIP-analysis showing the binding of c-Myc to COUP-TFII promoter and c-Myc and Smad4 to Cyclin D1 promoters in Pten ${ }^{-/-}$and CDCP1 Pten ${ }^{-1-}$ MEFs. Normal mouse IgC serves as negative control $(n=2)$. Error bars indicate SD. ${ }^{*} P<0.05 ;{ }^{* *} P<0.01$. Statistical test: 2 -tailed $t$ test.

demonstrating that PTEN loss leads to reciprocal feedback inhibition of AR activity (47). Thus, inhibition of PI3K leads to increased AR levels that promote the following downregulation of CDCP1.

CDCP1 targeting improves the efficacy of ADT. Given that androgen deprivation conditions elevate CDCP1 expression in ADS tumor cell lines, we postulated that compounds that block or degrade CDCP1 could be ideally used in combination with ADTs to prevent the emergence of ADI prostate tumor cells. To assess this hypothesis, we used the anti-CDCP1 monoclonal antibody CUB4, which binds the N-terminal domain of human CDCP1 and promotes CDCP1 internalization and degradation (27). Cotreatment of LNCaP cells with CUB4 and enzalutamide strongly affected the proliferation of these cells by inducing senescence. In contrast, enzalutamide-untreated cells were only slightly affected by the anti-CDCP1 antibody due to the low basal levels of CDCP1 in LNCaP cells (Supplemental Figure 8G). We next reasoned that tumor cell eradication rather than senescence induction could be a preferable outcome of CDCP1 targeting therapies (49). Therefore, we developed an anti-CDCP1 IL carrying doxorubicin to eliminate CDCP1-overexpressing prostate tumor cells induced by the enzalutamide treatment. Note that the anti-CDCP1 IL was generated by using the FAB of the CUB4 antibody (27). To allow the selective delivery of doxorubicin to the tumor cells, the antiCDCP1 ILs were designed with a size of $120 \mathrm{~nm}$. This size allows the preferential delivery of immunoliposome in tumor tissues due to the enhanced permeability and retention (EPR) effect of the cancer blood vessels (50-52).

Enzalutamide treatment, in combination with anti-CDCP1 ILs, induced a strong apoptotic response and blocked the emergence of $\mathrm{CDCP} 1^{+} \mathrm{ADI}$ cells in a time-course experiment (Figure $6, \mathrm{~A}$ and $\mathrm{B})$. In line with the previous experiments, treatment with anti-CDCP1 ILs affected the proliferation of LNCaP cells only in the presence of enzalutamide treatment. To validate these results in vivo, LNCaP cells were injected subcutaneously into SCID mice and upon the establishment of tumors, mice were treated with enzalutamide $(10 \mathrm{mg} / \mathrm{kg})$ with or without anti-CDCP1 ILs. Although enzalutamide showed minor effects on tumor growth, the combination of enzalutamide and anti-CDCP1 ILs significantly affected tumor growth (Figure 6, C and D). Note that Western blot analysis showed a significant increase in the levels of CDCP1 upon enzalutamide treatment in vivo, which was abolished upon combination treatment (Supplemental Figure 8H). Together these data suggest that CDCP1 targeting agents are effective when used in combination with $\mathrm{ADT}$.

\section{Discussion}

The present study highlights the crucial role of CDCP1 in promoting prostate cancer tumorigenesis and progression, and its ability to drive metastasis and CRPC in cooperation with PTEN deficiency. Since CDCP1 is highly expressed in MCRPC patients and can be easily targeted, our work opens new opportunities for combinatorial therapies. PTEN is one of the most frequently altered tumor suppressor genes in human prostate cancer, whereas complete loss of PTEN is frequently observed in metastatic prostate cancer (7). Previous evidence demonstrated that Pten $^{\mathrm{pc}-/-}$ develops indolent tumors characterized by a senescence response that, acting as an intrinsic barrier, constrain prostate cancer progression (11, 12). However, the mechanism by which PTEN-null benign tumors acquire metastatic potential remained poorly understood (12-14). CDCP1, a transmembrane protein that acts as a substrate for SRC family kinases, is overexpressed in a variety of tumors and has been associated with cancer development, invasion, and metastasis $(20,21)$. Although CDCP1 has been considered as an oncogene, recent publications demonstrate that CDCP1 inactivation accelerates mammary and skin tumorigenesis in the PyMT and SmoM2 models, respectively (25). As recently demonstrated, loss of CDCP1 can change the spectrum of SRC substrate phosphorylation in cells kept in suspension. Indeed, CDCP1 negatively regulates c-SRC and PKCd in suspended cells by sequestering these kinases away from their canonical substrates. As a consequence, SRC can phosphorylate CDK5R1/p35, thereby triggering the loss of ITGB1/b1-integrin inside-out activation (22).

In prostate cancer, the role of CDCP1 remains poorly characterized due to the lack of an in vivo model. Previous reports demonstrate that CDCP1 overexpression increases cellular proliferation in 2 human prostate cancer cell lines with validation of its elevated expression in a limited number of primary prostate tumor samples $(28,53)$. In an attempt to clarify the function of CDCP1 in prostate cancer, we generated the first prostate-specific CDCP1-overexpressing transgenic mouse model and assessed the level of CDCP1 in different prostate cancer TMAs, including more than 990 cases spanning benign, primary, and metastatic prostate cancer. We demonstrated that CDCP1 is overexpressed in a subset of advanced and metastatic prostate cancers, where it is frequently associated with loss of PTEN. Moreover, we showed in vivo that CDCP1 cooperates with PTEN loss to promote the emergence of metastases and CRPC through the upregulation of the MAPK pathway. Previous evidence demonstrates that patients who develop resistance to ADT present tumors with elevated levels of MAPK pathway and that activation of the MAPK pathway cooperates with PTEN deficiency to promote $\operatorname{mCRPC}(8,54)$. Mechanistically, we 
A

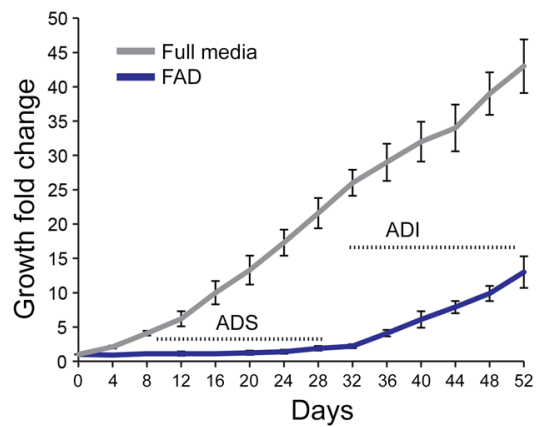

B

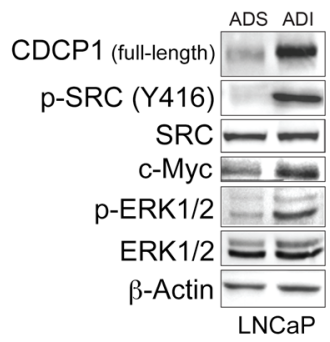

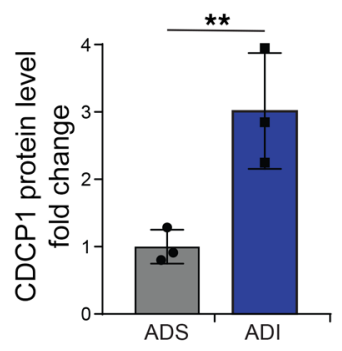
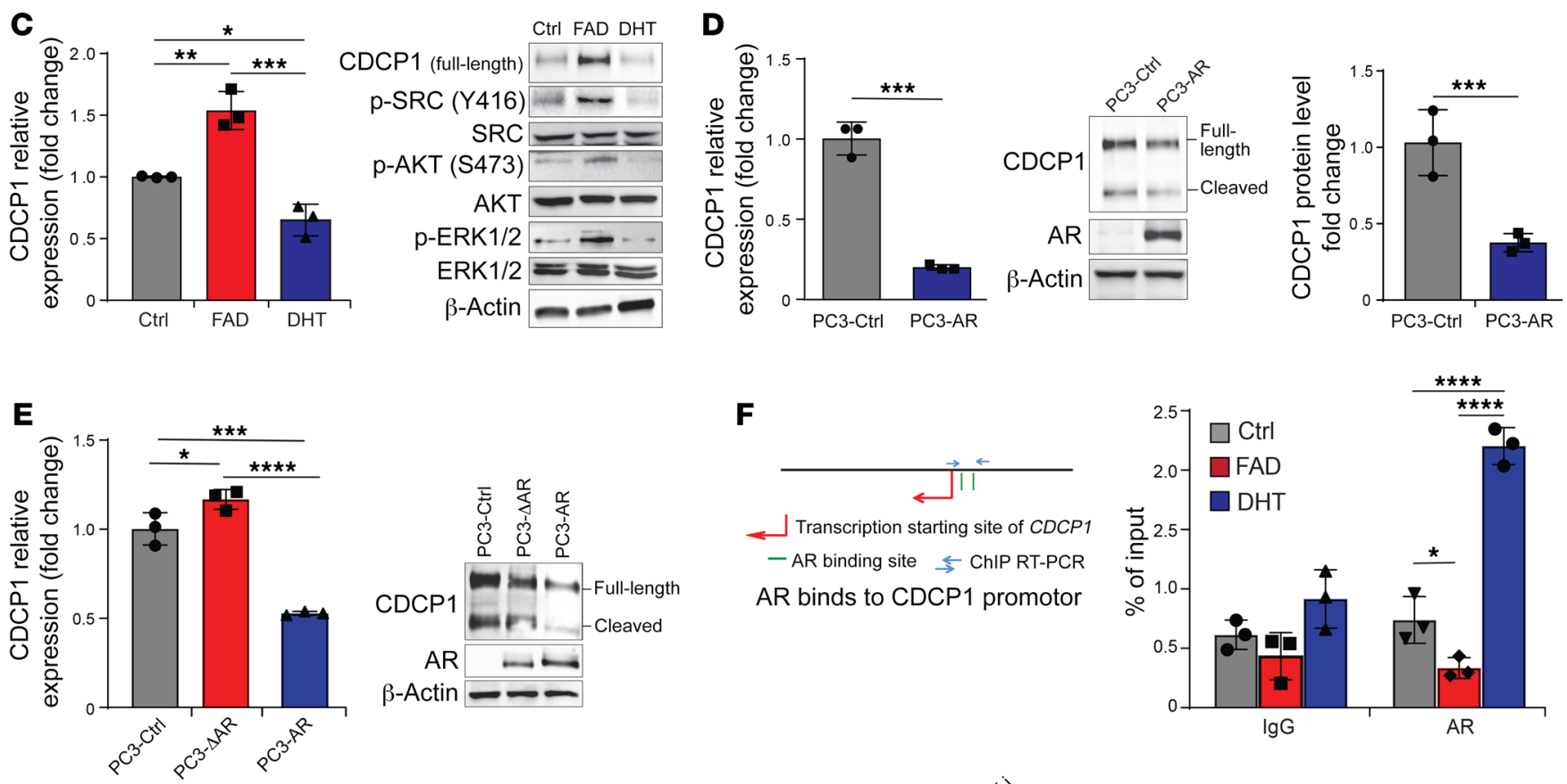

$\mathbf{F}$
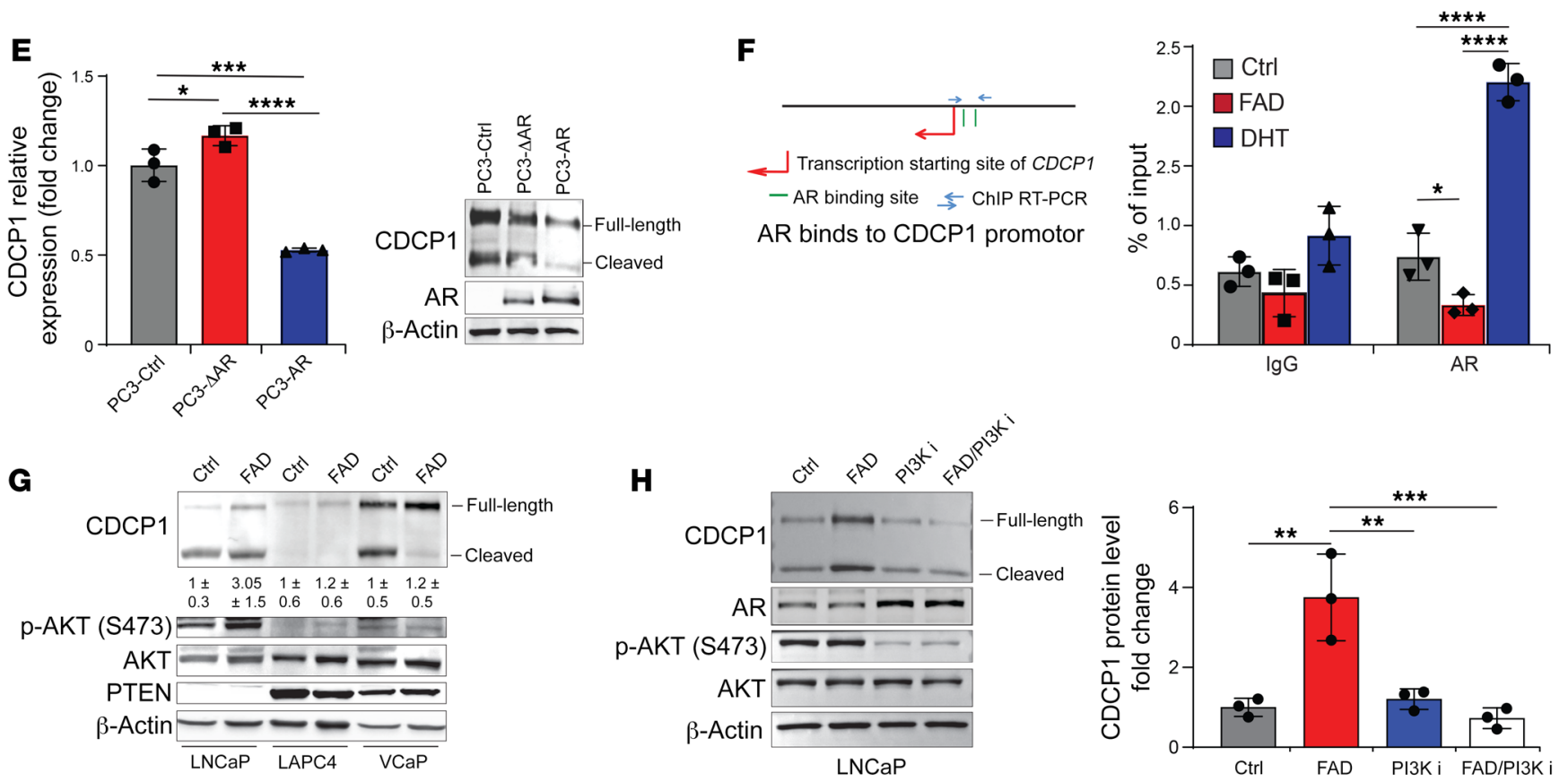

Figure 5. Androgen deprivation in human tumor samples and cells induces CDCP1 expression. (A) Quantification of fold change in growth by crystal violet in LNCaP cell line grown in full media and in FAD. Dotted lines indicate ADS and ADI phases $(n=3)$. (B) Western blot analysis of indicated proteins in LNCaP-ADS and LNCaP-ADI. Quantification of fold change in CDCP1 protein levels in LNCaP-ADS and LNCaP-ADI $(n=3)$. (C) qRT-PCR analysis of CDCP1 mRNA levels in LNCaP grown in full media; FAD and stimulated with dihydrotestosterone (DHT, $1 \mu \mathrm{M}$, 16 hours) after being grown for 2 days in FAD. Western blot analysis of indicated proteins in LNCaP grown under the described conditions $(n=3)$. (D) qRT-PCR of CDCP1 mRNA levels in PC3 expressing empty vector (PC3-Ctrl) and in PC3 overexpressing full-length androgen receptor (PC3-AR). Western blot analysis and protein fold change quantification of indicated proteins in PC3-Ctrl and PC3-AR cell lines $(n=3)$. (E) qRT-PCR and Western blot analysis in PC3-Ctrl, PC3-AR, and PC3 overexpressing DNAbinding mutant of AR (PC3- $\triangle \mathrm{AR}$ ) of CDCP1 mRNA and indicated proteins $(n=3)$. (F) Scheme represents the AR binding site on CDCP1 promoter. qRT-PCR of ChIP-analysis showing the binding of AR to CDCP1 promoter in LNCaP cell line grown in full media; FAD after DHT stimulation. Normal mouse IgC served as a negative control $(n=3)$. (C) Western blot analysis of indicated protein in LNCaP, LAPC4, and VCaP kept in normal conditions and in FAD ( $n=3)$. (H) Western blot analysis of indicated proteins in LNCaP treated with PI3K inhibitor in normal conditions or in FAD. Quantification of fold change in CDCP1 protein levels in LNCaP untreated or treated with PI3K inhibitor in normal conditions and in $\operatorname{FAD}(n=3)$. Error bars indicate $\mathrm{SD}$. ${ }^{*} P<0.05 ;{ }^{* *} P<0.01$; ${ }^{* *} P<0.001 ;{ }^{* * *} P<0.0001$. The following statistical tests were used: 1-way ANOVA adjusted for multiple comparisons using Tukey's test for $\mathbf{C}, \mathbf{E}, \mathbf{F}$, and $\mathbf{H}$, and unpaired 2-tailed $t$ test for $\mathbf{B}$ and $\mathbf{D}$. 
A

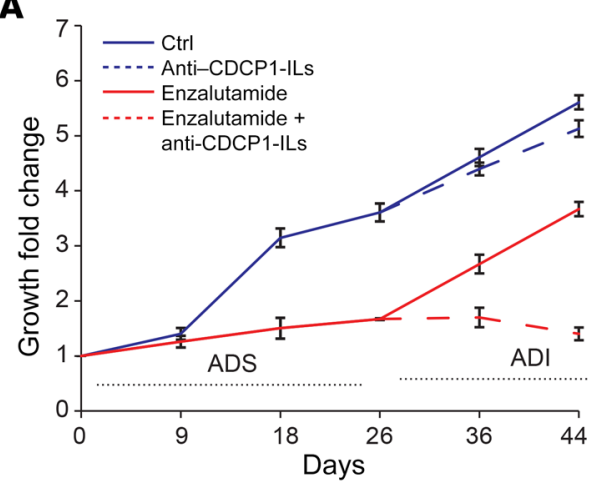

C

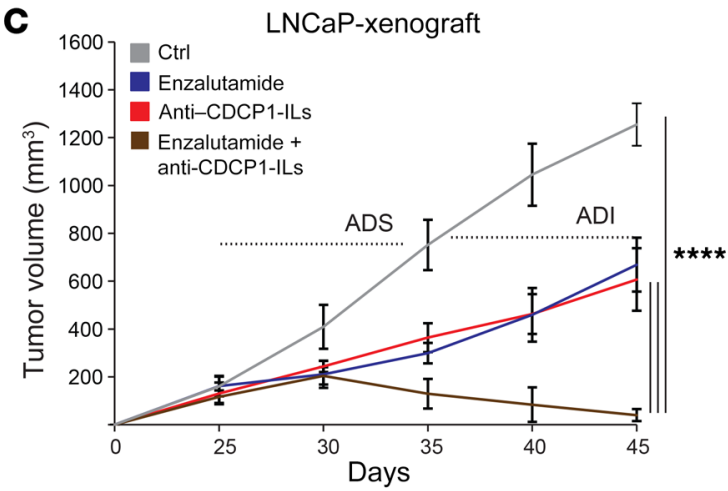

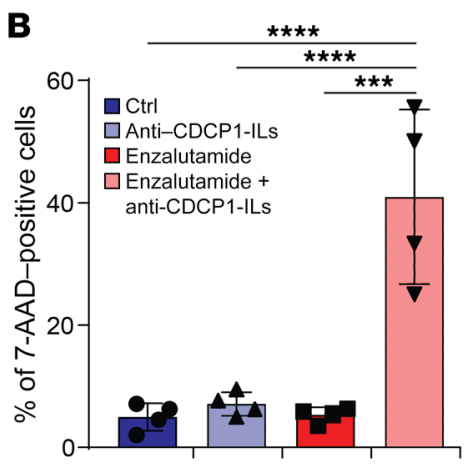

D
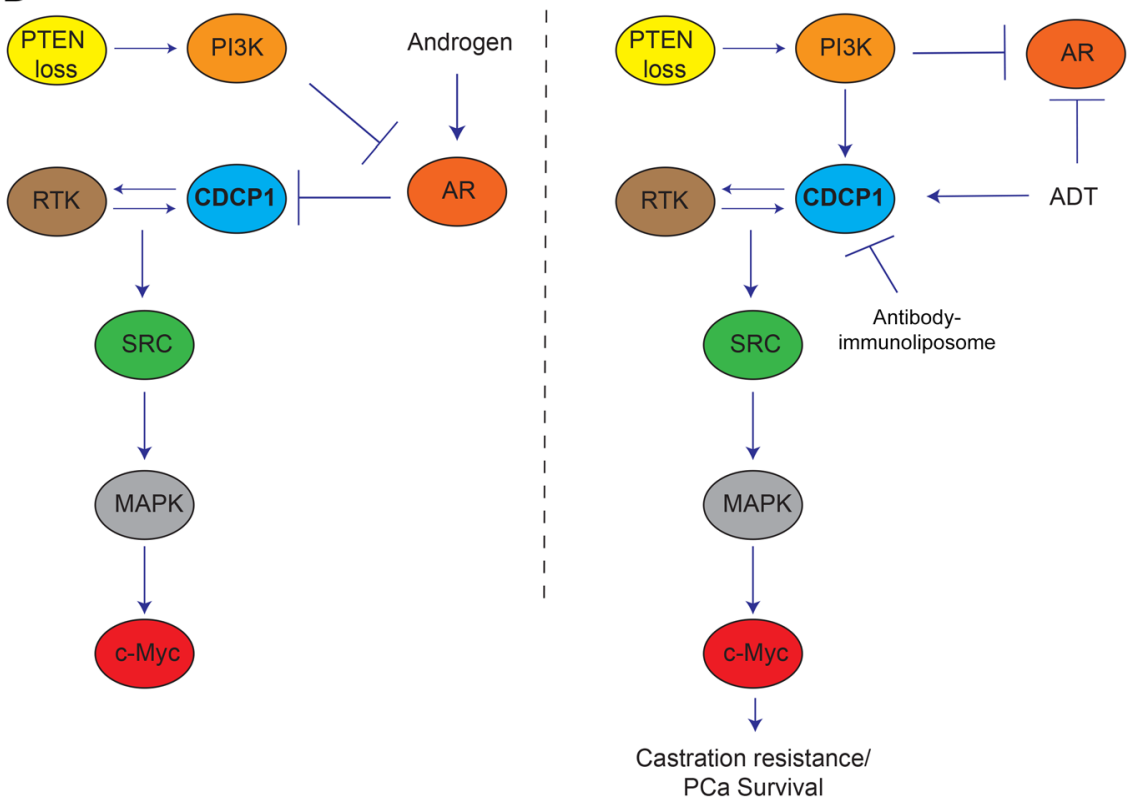

Figure 6. CDCP1 targeting improves the efficacy of ADTs. (A) Quantification of fold change in growth by crystal violet in LNCaP cell line untreated and treated with enzalutamide $(10 \mu \mathrm{M})$ in combination with or without the immune-liposome (anti-CDCP1-ILs). Enzalutamide (10 $\mu \mathrm{M})$ treatment lasted for 26 days. After that, cells were treated in combination with anti-CDCP1-ILs. (B) Quantification of cell death with 7-AAD staining in cells untreated and treated with enzalutamide in combination with or without the anti-CDCP1-ILs $(n=4)$. (C) Xenografts tumor growth $\left(\mathrm{mm}^{3}\right)$ of LNCaP cell line untreated or treated with enzalutamide in the presence or absence of the anti-CDCP1-ILs. Upon tumor establishment, mice were treated with enzalutamide (10 mg/kg) for 45 days. After the first week of enzalutamide treatments, mice were divided into 2 groups and treated with or without the anti-CDCP1-ILs. Note that the 2 treatments were consecutive $(n=4)$. (D) The model represents the role of CDCP1 in CRPC. PI3K partially blocks AR. ADT blocks AR completely and upregulates CDCP1 levels. Error bars indicate SD for $\mathbf{B}$ and SEM for $\mathbf{C}$. ${ }^{* *} P<0.001 ;{ }^{* * *} P<0.0001$. The following statistical test was used: 1-way ANOVA adjusted for multiple comparisons using Tukey's test.

showed that CDCP1 overexpression increases c-Myc levels in a Src-dependent manner. This, in turn, promotes the activation of COUP-TFII that further inhibits Smad4-dependent transcription. As a result, Cyclin D1 gets upregulated and CDCP1 Pten $^{\mathrm{pc}-/-}$ tumors bypass senescence and progress toward a metastatic phenotype.

Of note, we found that CDCP1 mRNA and protein levels increase in PTEN-deficient cells treated with enzalutamide, a standard of therapy for CRPC patients. Finally, we provided evidence that the AR can suppress the transcription of CDCP1 in particular in cells carrying the loss of PTEN (Figure 5E). Overexpression of the $\mathrm{AR}$ in $\mathrm{AR}^{-}$prostate cancer cell lines significantly decreased CDCP1 levels, supporting our observations. The reciprocal feedback regulation of PI3K and androgen receptor signaling in PTEN-deficient prostate cancer can explain the observed
PTEN-CDCP1 dependency (47). Although we did not formally prove it, we believe that AR mutations, AR splicing variants, and the AR rewiring may also account for the upregulation of CDCP1 observed in metastatic prostate cancer patients not treated with ADT due to the lack of AR binding to the CDCP1 promoter. Therapeutically, we demonstrated that CDCP1 inhibition, in combination with $\mathrm{ADT}$, might represent an interesting new therapeutic approach in prostate cancer. Indeed, we showed that inhibition of CDCP1 in combination with enzalutamide has the potential for prostate cancer treatment. Treatment of PTEN-deficient human prostate tumor cells with enzalutamide promoted the upregulation of CDCP1 levels. This treatment rendered PTEN-null cells more sensitive to CDCP1 targeting agents. On the other hand, enzalutamide-untreated cells did not respond to CDCP1-targeting agents. 


\section{Table 5. Mouse primers for real-time PCR}

\begin{tabular}{|c|c|}
\hline Primer & Sequence \\
\hline p16Ink4a forward & 5'-CGCAGGTTCTTGGTCACTGT-3' \\
\hline p16Ink4a reverse & 5'-TGTTCACGAAAGCCAGAGCG-3' \\
\hline p21 forward & 5'-GGGCGCACGATGTTCAGAA-3' \\
\hline p21 reverse & 5'-CACCACCAGGTCGAAATGGG-3' \\
\hline p27 forward & 5'-GCAAAACAAAAGGGCCAACA-3 \\
\hline p27 reverse & 5'-GGGCGTCTGCTCCACAGT-3' \\
\hline Gapdh forward & 5'-AGGTCGGTGTGAACGGATTTG-3 \\
\hline Gapdh reverse & 5'-TGTAGACCATGTAGTTGAGGT-3' \\
\hline Rn185 forward & 5'-ACCGCAGCTAGGAATAATG-3' \\
\hline Rn18S reverse & 5'-GCCTCAGTTCCGAAAACCA-3' \\
\hline COUP-TFIl forward & 5'-TCAACTGCСACTCGTACCTG-3' \\
\hline COUP-TFII reverse & 5'-CATGATGTTGTTAGGCTG-3' \\
\hline Cyclin D1 forward & 5'-GCGTACCCTCACACCAATC-3' \\
\hline Cyclin D1 reverse & 5'-СТССТСТTСССАСТTСТССТС-3' \\
\hline c-Myc forward & 5'-CTGGACCAGGGAGTGGAGT-3' \\
\hline c-Myc reverse & 5'-ACGTAGTAGTCGGTTCTCA-3' \\
\hline
\end{tabular}

Moreover, we demonstrated in vivo that enzalutamide, in combination with a new CDCP1 immunoliposome carrying doxorubicin, significantly inhibits tumor progression, inducing a strong apoptotic response. These findings demonstrate that CDCP1-targeting therapies should be combined with ADT to maximize the efficacy of this standard of treatment. Therapeutically, the use of an antiCDCP1 IL containing doxorubicin has several advantages. First, liposomes loaded with doxorubicin are already in the clinic and are well tolerated by cancer patients. Second, the size of anti-CDCP1 IL allows its extravasation and accumulation preferentially at the tumor site due to the EPR effect (50-52). Third, the conjugation of the liposomes with the human FAB of the CDCP1 antibody increases the specificity and permanence of the IL in tumors overexpressing CDCP1, increasing its anticancer efficacy. On a negative side, since the anti-CDCP1 ILs have been generated with an antibody that recognizes human $\mathrm{CDCP} 1$, our experiments in mice cannot exclude the risk of systemic toxicities of this IL, and further experiments should be carried on by using a mouse antibody.

\section{Methods}

Acquisition of MEFs. Primary MEFs were prepared as described previously from individual embryos of various genotypes (12). Briefly, MEFs for all genotypes were obtained by crossing male WT and Pten ${ }^{\text {lox-lox }}$ with female $C D C P 1^{\text {lox-stop-lox }}$ mice. Pregnant mice were sacrificed by cervical dislocation 13 or 14 days postcoitum. Embryos were harvested and the individual MEFs were cultured in DMEM containing 10\% fetal bovine serum and $1 \%$ penicillin-streptomycin. Primary Pten ${ }^{l o x} /$ lox MEFs were infected with retroviruses expressing either pMSCV-CRE-PUROIRES-GFP or pMSCV-PURO-IRES-GFP for 48 hours and selected with puromycin at a concentration of $3 \mu \mathrm{g} / \mathrm{mL}$ and as previously described. All mice were maintained under specific pathogen-free conditions in the animal facilities of the Institute for Research in Biomedicine. Experiments were performed according to state guidelines and approved by the local ethical committee.

Cell culture and reagents. Human prostate carcinoma cell lines were purchased from ATCC and maintained according to the suppli- er's recommendation. Cells were transduced with PLKO or TRIPZ doxycycline-inducible lentiviral construct, against human CDCP1 gene or empty vector, obtained by Thermo Fisher Scientific (clones V3THS_329377 and V2THS_191307). LNCaP-abl and LAPC4 cells were a gift from Jean-Philippe Theurillat (Institute of Oncology Research, Bellinzona, Switzerland). PC3-ARs were generated by infecting them with retroviruses expressing full-length human AR (provided by JeanPhilippe Theurillat). PC3- $\triangle$ ARs were generated using the expression of human AR with the deletion of amino acids 538 to 614, deletion of AR DNA binding domain (Addgene, catalog 89107). LNCaP-ADI cells were generated from parental LNCaP by growing them in RPMI 1640 containing 10\% charcoal-stripped FBS. Androgen stimulation experiments were performed using $1 \mathrm{nM}$ of the $5 \alpha$-DHT (MilliporeSigma, catalog 521-18-6). The FAD experiment was performed by culturing the cells in RPMI with charcoal-stripped FBS and enzalutamide. Enzalutamide (APExBIO, catalog A3003) was dissolved in DMSO at a concentration of $10 \mu \mathrm{M}$. The following antibodies were used for Western blotting: TagMyc (BD Pharmingen, catalog 551101; 1:1000); PTEN (Cell Signaling Technology, catalog 9552S; 1:1000); HSP90 (Cell Signaling Technology, catalog 4877S; 1:1000); c-Myc (Santa Cruz Biotechnology, catalog A713(G-4), 1:500); p21 (Abcam, catalog ab107099, 1:1000); $\beta$-actin (MilliporeSigma, catalog A5316; 1:5000); Cyclin D1 (Cell Signaling Technology, catalog 2978S, 1:1000); COUP-TFII (Perseus Proteomics, catalog PP-H7147-00; 1:1000); SMAD4 (Santa Cruz Biotechnology, catalog E0615; 1:500); p-SRC-Tyr416 (Cell Signaling Technology, catalog 6943S; 1:1000); SRC (Cell Signaling Technology, catalog 2123S; 1:1000); AKT (Cell Signaling Technology, catalog 9272S; 1:1000); p-AKT-S473 (Cell Signaling Technology, catalog 9171S; 1:1000); p53 (Abcam, catalog ab131442; 1:1000); CDCP1 (Cell Signaling Technology, catalog 4115; 1:1000); Erk1/2 (Cell Signaling Technology, catalog 4695S; 1:1000); p-Erk1/2-T202/Y204 (Cell Signaling Technology, catalog 4370S; 1:1000); S6 (Cell Signaling Technology, catalog 2317S; 1:1000); p-S6-Ser235/236 (Cell Signaling Technology, catalog 4857; 1:1000); AR (N-20) (Santa Cruz Biotechnology, catalog SC-816; 1:500). For IHC the following antibodies were used: Ki-67 (Thermo Fisher Scientific, clone SP6, catalog RM-9106-R7; rabbit polyclonal; unmasked water bath $98^{\circ} \mathrm{C}, \mathrm{pH} 6,20$ minutes; Lab Vision dilution ready to use); CDCP1 (Cell Signaling Technology, catalog 4115, rabbit polyclonal; unmasked water bath $98^{\circ} \mathrm{C}, \mathrm{pH} \mathrm{6,} 20$ minutes; 1:50); p-HP1 $\gamma$-Ser83 (Cell Signaling Technology, catalog 2600 , unmasked water bath $98^{\circ} \mathrm{C}$, pH 6, 20 minutes; 1:50); Cyclin D1 (Cell Signaling Technology, catalog

\section{Table 6. Human primers for real-time PCR}

\begin{tabular}{|c|c|}
\hline Primer & Sequence \\
\hline p21 forward & 5'-TGTCCGTCACAACCCATGC-3' \\
\hline p21 reverse & 5'-AAAGTCCAAGTTCCATCCCTC-3' \\
\hline p27 forward & 5'-TAATTGGGGCTCCGGCTAACT-3' \\
\hline p27 reverse & 5'- TGCAGGTCGCTTCCTTATTCC-3' \\
\hline GAPDH forward & 5'-AАТСССАТСАССАТСТTССА-3' \\
\hline GAPDH reverse & 5'-TCGACTCCACGACGTACTCA-3' \\
\hline C-MYC forward & 5'-CGGAACTCTTGTGCGTAAGG-3' \\
\hline$c-M Y C$ reverse & 5'-CTCAGCCAAGGTTGTCAGGT-3' \\
\hline$C D C P 1$ forward & 5'-TCGTTCCACCCCAGAAATGT-3' \\
\hline$C D C P 1$ reverse & 5'-CATCATGCACACACGTTTTAT-3' \\
\hline
\end{tabular}


2978S); AR (N-20) (Santa Cruz Biotechnology, catalog SC-816, rabbit polyclonal; unmasked water bath $98^{\circ} \mathrm{C}, \mathrm{pH}$ 6, 20 minutes; 1:300); wide spectrum cytokeratin (pankeratin) (DAKO, catalog Z0622; rabbit polyclonal; unmasked water bath $98^{\circ} \mathrm{C}, \mathrm{pH} 9,20$ minutes; 1:2000). For IF, the following antibodies were used: E-cadherin (BD Biosciences, clone 26, catalog 610181; mouse monoclonal; unmasked water bath $98^{\circ} \mathrm{C}, \mathrm{pH}$ 9, 20 minutes; 1:700); CK5 (Abcam, catalog ab52635; rabbit polyclonal; unmasked water bath $98^{\circ} \mathrm{C}, \mathrm{pH} 9,20$ minutes; 1:500); CK8 (Abcam, catalog ab59400; rabbit polyclonal; unmasked water bath $98^{\circ} \mathrm{C}, \mathrm{pH} 9,20$ minutes; 1:150); and CDCP1 (Cell Signaling Technology, catalog 4115, 1:100). The E-cadherin antibody (Developmental Studies Hybridoma Bank [DSHB], DCAD2, 1:100) was used for the drosophila experiment in Supplemental Figure 3. c-Myc siRNA and negative control siRNA were purchased from MilliporeSigma (catalog 8024873724-000050 and 8024873724-000060). The cells were transfected with Lipofectamine RNAiMAX (Invitrogen, catalog 13778-030) according to the manufacturer's protocol.

Generation of GAL4-UAS-CDCP1-WT and GAL4-UAS-CDCP1-delta Drosophila melanogaster lines and immunofluorescence. UAS-egfr.B (5368), src64BP1 (7379), Src42AK10108 (10969), GMR-gal4 (1104), and ptc-gal4 (2017) lines were obtained from the Bloomington Drosophila Stock Centre. Cultures were carried out on a cornmeal/agar diet, $(6.65 \%$ cornmeal, $7.15 \%$ dextrose, $5 \%$ yeast, $0.66 \%$ agar supplemented with $2.2 \%$ nipagin and $3.4 \mathrm{~mL}$ propionic acid) and maintained at $25^{\circ} \mathrm{C}$ and $29^{\circ} \mathrm{C}$. To overexpress human CDCP1-WT and CDCP1delta, UAS transgenic lines were generated from human CDCP1-WT and CDCP1-delta cDNA with the following primer pair: $5^{\prime}$-GATATCCACCATGGCCGGCCTGAACTGCGGG-3' and 5'-ACTAGTTCAATGGTGATGGTGATGATG-3'. PCR was performed with Q5 high-fidelity polymerase (New England Biolabs, catalog M0491S). PCR products were cloned using the Zero Blunt TOPO PCR Cloning Kit (Life Technologies, catalog K2800-20) before cloning into the pUAST-attB vector. The constructs were sequence-verified and the transgenic lines established through PhiC31 integrase-mediated transgenesis (BestGene, attP site VK27). Salivary glands were dissected in PBS, fixed in $4 \%$ paraformaldehyde (PFA) in PBS, washed in PBT (PBS containing $0.1 \%$ Triton $\mathrm{X}-100$ ), and incubated with primary antibodies in PAXDG (PBS containing 1\% BSA, 0.3\% Triton X-100, 0.3\% deoxycholate, and $5 \%$ goat serum) overnight at $4^{\circ} \mathrm{C}$. Tissues were washed with PBT, incubated with secondary antibodies in PAXDG for 5 hours at $4^{\circ} \mathrm{C}$, and mounted in Vectashield mounting media (Vector Laboratories). Alexa Fluor 568-8-conjugated anti-rabbit and Alexa Fluor 488-conjugated anti-rat antibodies were used as secondary antibodies (Molecular Probes). Images of adult eye and bristle were taken with a Leica M165 FC microscope equipped with SXY-I30 3M Pixel Color Camera. Fluorescent images of salivary glands were taken with Leica M165 FC fluorescent microscope equipped with Leica DFC $3000 \mathrm{G}$ digital camera.

$C D C P 1$ protein expression in human prostate cancer. The first group of TMAs (Supplemental Figure 1A) was composed of 2 TMAs. The first TMA included benign prostate tissue and prostate cancer at different stages ( $n=237$ ), as previously reported (32). Spots with metastases were not included in the analysis, to avoid false-negative results due to poor fixation of tissue (mostly material from autopsies). The second TMA ( $n=192)$ consisted of locally advanced, inoperable, mostly metastatic prostate cancer including CRPC and hormone naive (untreated) prostate cancer, as previously reported (32). For distant metastasis, CDCP1 staining was performed on 6 regular histological sections of distant and lymph node prostate cancer metastases. The second group of TMAs (Figure 1, A-D, and Tables 1-4) was composed of 3 different TMAs as previously described $(30,31)$. Briefly, the first TMA included $201 \mathrm{BHP}$, RPE, CRPC, and metastasis samples. The second TMA included 323 PCa samples of TUP-P and RPE. The third TMA included 82 CRPC samples. To determine $\mathrm{H}$ score, the intensity of membrane CDPC1 staining (on a scale of 0 [no staining], 1+ [weak staining], 2+ [moderate staining], and $3+$ [strong staining]) was multiplied by the percentage of positive tumor cells. In the second group of TMAs, PTEN status was determined by FISH or IHC analysis as previously described (30). The use of the clinical samples for TMA construction was approved by the ethics committee of the University of Basel and the University of Zürich. For paired diagnostic (HSPC) and CRPC biopsies (Supplemental Figure 1B), patients were identified from a population of men with mCRPC treated at the Royal Marsden NHS Foundation Trust. All patients provided written informed consent and were enrolled in institutional protocols approved by the Royal Marsden NHS Foundation Trust Hospital (London, United Kingdom) ethics review committee (reference no. 04/Q0801/60). Twenty-five patients with a diagnosis of prostate adenocarcinoma with sufficient formalin-fixed, paraffinembedded (FFPE), matched diagnostic (archival) hormone-sensitive prostate cancer (HSPC), and CRPC tissue for CDCP1 immunohistochemistry were selected. HSPC tissue demonstrated adenocarcinoma and was obtained from either prostate needle biopsy (21 cases), transurethral resection of the prostate (TURP; 3 cases), or bone biopsy (1 case). CRPC tissue was obtained from the same patients through biopsies of bone (19 cases), lymph node ( 5 cases), or liver (1 case). All tissue blocks were freshly sectioned and only considered for IHC analyses if adequate material was present ( $\geq 50$ tumor cells).

Bioinformatic analysis. Correlation between CDCP1 and PTEN in prostate cancer data sets $(5,54-57)$ was carried out using Spearman's correlation, which estimates a correlation coefficient value $R$ and a significant $\mathrm{P}$ value.

We retrieved gene expression and DNA methylation from The Cancer Genome Atlas (TCGA) and performed a correlation analysis between the mRNA expression level and the methylation profile of CDCP1 (Pearson correlation). Methylation level of CDCP1 was determined as the mean of $\beta$ values within a distance of about $1000 \mathrm{bp}$ from the transcription start site (TSS). Samples were classified into quartiles (Q1-Q4) based on mRNA expression level of CDP1 or according to its methylation. Dependency between CDCP1 expression and PTEN deletions/mutations was determined using $\chi^{2}$ test. Survival analysis was performed using the Kaplan-Meier estimator and Cox regression model.

Immunoblotting. Tissue and cell lysates were prepared with RIPA buffer (Cell Signaling Technology, catalog 9806) with PMSF (phenylmethanesulfonyl fluoride; MilliporeSigma, catalog 329-98-6). Protein concentrations of the lysates were measured by Pierce BCA Protein Assay Kit (Thermo Fisher Scientific, catalog 23225). The lysates were then resolved by SDS-PAGE and immunoblotted with the indicated antibodies. For analysis of fly tissue, wandering third-instar larvae were rinsed in PBS, and salivary glands were dissected out, washed in PBS, and homogenized in SDS sample buffer.

Real-time PCR. RNA was extracted using TRIzol Plus RNA Purification Kit (Life Technologies, catalog 12183555). Total RNA (1 $\mu \mathrm{g})$ was used for cDNA synthesis using SuperScript III Platinum One-Step qRTPCR Kit (Life Technologies, catalog 11732-020). Quantitative real-time PCR (qRT-PCR) was performed as previously described (12). Primers 
used are listed in Tables 5 and 6. All qRT-PCR data presented were normalized using GAPDH, HRPT, or 18S rRNA.

ChIP assay. Cells were cultured to a confluence of 90\%-95\% and were cross-linked with $1 \%$ formalin for 10 minutes followed by the addition of $2.5 \mathrm{M}$ glycine for 5 minutes at room temperature. The culture medium was aspirated and the cells were washed twice with ice-cold PBS. Nuclear extracts were sonicated using a Misonix 3000 model sonicator to sheer cross-linked DNA to an average fragment size of approximately $500 \mathrm{bp}$. Sonicated chromatin was incubated for 16 hours at $4^{\circ} \mathrm{C}$ on a rotor with $\gamma$-bind Plus sepharose beads (GE Healthcare, catalog 17-0886-01) conjugated with either anti-c-Myc ([9E10]x L0815) anti-SMAD4 ([B-8]; Santa Cruz, catalog E0615) or mouse-IgG antibody (Millipore, catalog 92590). After incubation, beads were washed thoroughly and then centrifuged. The chromatin was eluted from the beads, and cross-links were removed by incubation at $56^{\circ} \mathrm{C}$ for 12 hours. DNA was then purified using the QIAquick PCR Purification Kit (Qiagen, catalog 28104). The binding of the transcription factor, c-Myc, on Cyclin D1 promoter was determined using SABiosciences' proprietary database (DECODE, DECipherment of DNA Elements). The primer mixes used for ChIP assay in MEFs were as follows: (a) to detect Smad4 binding site (SBE) on Cyclin D1 promotor: SBEChIP forward 5'-CCGCTTAGTCCCCATTCTAAAG-3' and SBEChIP reverse $5^{\prime}$-GGCATCTCCATTCTTAATCCAG-3'; (b) to detect c-Myc binding on Coup-tfII promotor: COUP-TFII ChIP forward 5'-GTGCGGGGACAAGTCGAGCGG-3' and COUP-TFII ChIP reverse 5'-GCGGTGGTGCTGGTCGATGGG-3'; (c) to detect c-Myc binding on Cyclin D1 promotor: EpiTect ChIP qPCR Primer Assay For Mouse Ccnd1, NM_007631.2 (-)04 Kb (catalog GPM1053924(-)04A). The primer mix used for ChIP assay in LNCaP to detect AR binding site on CDCP1 promoter was: forward 5'-GAATTTGTCCTCGATTCAG-3' and reverse 5'-GCCAGAGGTCTGTTGGAC-3'. ChIP qPCR was performed using KAPA SYBR FAST ABI qPCR Master Mix solution (KAPA Biosystem, Roche, catalog 07959389001) on Step One Real-Time PCR systems (Applied Biosystems).

Proliferation and senescence assays. Proliferation assay in MEFs was performed by plating $10^{4}$ cells per well in a 24 -well plate in triplicate while that in human prostate cancer cell lines was performed by plating $1 \times 10^{4}-2 \times 10^{4}$ cells per well in a 24 -well plate in triplicate. Cell proliferation was monitored at days $0,3,6$, and 9 whereby cells were fixed for 15 minutes in a solution of $10 \%$ buffered formalin washed with PBS (pH 7.2) and subsequently stained with $0.01 \%$ Crystal violet solution. Excessive staining was removed by washing the plates with distilled water and by drying them overnight. Crystal violet-stained cells were dissolved in $10 \%$ acetic acid solution for 30 minutes on a shaker and the extracted dye was read with a spectrophotometer at $590 \mathrm{~nm}$. Cellular senescence in vitro was assessed using the Senescence $\beta$-Galactosidase Staining Kit (Cell Signaling, catalog 9860) as per the manufacturer's instructions and the quantification was done by counting the total number of cells with Hoechst 3342, trihydrochloride, trihydrate (Invitrogen; catalog 953557).

Liposomes formulation. Stealth liposomes (SLs) were prepared using $\mathrm{HSPC} / \mathrm{CHOL} / \mathrm{mPEG}_{5 \mathrm{kD}}$-DSPE at a molar ratio of 18:9:1. The lipid film, obtained by evaporating a chloroform solution of the components, was hydrated with a solution of $250 \mathrm{mM}$ ammonium sulfate (pH 5.5) and then extruded at $60^{\circ} \mathrm{C}$ until reaching the vesicle size of approximately $100 \mathrm{~nm}$. The external buffer was exchanged to $\mathrm{PBS} \mathrm{pH}$ 7.4 by a PD-10 desalting column. Doxorubicin (DXR) was encapsulat- ed by remote loading (DXR/HSPC 0.2:1 wt/wt) at $60^{\circ} \mathrm{C}$. Free DXR was removed using a PD-10 desalting column and the drug loading was determined spectrophotometrically $(\lambda=477 \mathrm{~nm})$ in methanol. The CUB4 Fab'-coupled PEG-phospholipid derivative was prepared by reacting the Fab' of CUB4, obtained by enzymatic digestion of Fc and Fab2 reduction as described below, with maleimide-PEG-DSPE. The synthetized CUB4 Fab'-PEG-DSPE was then introduced on the liposome surface by the post-insertion technique, described below, to provide stealth immunoliposomes (SILs). Briefly, CUB4 was enzymatically digested with pepsin (1:50 wt/wt enzyme/substrate, 3 hours at $37^{\circ} \mathrm{C}$ ) in $0.1 \mathrm{M}$ sodium acetate at $\mathrm{pH} 3.8$, followed by FPLC analysis on a Superose 12 10/300 GL column using PBS pH 7.4 (flow-rate $0.5 \mathrm{~mL} / \mathrm{min}$ ). The $\mathrm{F}\left(\mathrm{ab}^{\prime}\right)_{2}$ fragment was collected and treated for 30 minutes at room temperature with $10 \mathrm{mM}$ cysteamine to yield the Fab' fragment, following purification by FPLC using $50 \mathrm{mM}$ phosphate buffer, $150 \mathrm{mM} \mathrm{NaCl}$, and $10 \mathrm{mM}$ EDTA, pH 5. By exploiting its free sulfhydryl groups, Fab' was immediately coupled (overnight at room temperature, $\mathrm{pH}$ 7.0-7.5) to the maleimide groups of mixed micelles composed of maleimide- $\mathrm{PEG}_{5 \mathrm{kDa}}$-DSPE/ $\mathrm{mPEG}_{5 \mathrm{kDa}}-\mathrm{DSPE} 4: 1 \mathrm{~mol} / \mathrm{mol}$ at a final molar ratio of 10:1 maleimide/Fab'. In the last step, the Fab'$\mathrm{PEG}_{5 \mathrm{kDa}}-\mathrm{DSPE}: \mathrm{MPEG}_{5 \mathrm{kDa}}-\mathrm{DSPE}$ micelles were inserted on SL surface (post-insertion technique) by incubation of these micelles with SL for 1 hour at $60^{\circ} \mathrm{C}$ at a molar ratio of 0.05:1 PEG/HSPC to achieve SIL, which were purified on a Sepharose CL-4B column using PBS pH 7.4 and Fab' quantification by BCA assay.

Statistics. All data points are presented for quantitative data, with an overlay of the mean with SD and SEM (specified in the figure legends). All statistical analysis were performed using Graph Pad Prism 8 or Microsoft Excel 2016 or R-studio. A 1- or 2-tailed Student $t$ test was used for statistical analysis (as specified in the figure legends). Other methods of statistical analysis are indicated in the figure legends.

Study approval. All mice were maintained under specific pathogen-free conditions in the animal facilities of the Institute for Research in Biomedicine, in Bellinzona, Switzerland. Experiments were performed according to state guidelines and approved by the local ethics committee. The Pten ${ }^{\text {loxP }}$ conditional knockout mice were previously described (12). CDCP1 conditional overexpression was generated as described in the text. However, to check for correct targeting of the transgene, DNAs from different clones were digested with SpeI and analyzed for correct targeting using an internal 840-bp PstI/XbaI the ColA1 3'probe that hybridized also with the WT allele (33). To obtain the prostate-specific overexpression of CDCP1 and deletion of Pten, female CDCP1 and/or Pten ${ }^{\text {loxP/loxP }}$ mice were crossed with male Probasin-Cre4 (Pb-Cre4) transgenic mice (34). To sheer cross-linked DNA to an average fragment for genotyping, tail-derived DNA was subjected to PCR analyses. For Pten ${ }^{\text {loxP/loxp }}$ genotyping, primer 1 (5'-AAAAGTTCCCCTGATGATGATTTGT-3') and primer 2 (5'-TGTTTTTGACCAATTAAAGTAGGCTGTG-3') were used. For detecting the allele in the prostate, primer 3 (5'-TTCTCTTGAGCACTGTTTCACAGGC-3') and primer 1 were used. For Pb-Cre 4 , primer 1 (5'-TGATGGACATGTTCAGGGATC-3') and primer 2 (5'-GCCACCAGTCTGCATGA-3') were used. For CDCP1 mice, primer 1 (5'-CAAGGGAGAAGAGAGTGCGG-3') and primer 2 (5'-CCCAACAATGGGGATGTAAG-3') were used, both for genotyping and detecting the allele in the prostate. For the downregulation of CDCP1, cells were infected with PLKO-shCDCP1 and doxycycline-inducible pTripz-CDCP1-shRNA. As control for both vectors, we used nontarget shRNA. In the xenograft experi- 
ments, $1 \times 10^{6}$ Tripz-shCDCP1 or Tripz-shRNA controls, PC3 cells, and $1 \times 10^{6} \mathrm{LNCaP}$ cells were injected s.c. in SCID-NOD mice. After tumor cell injection, tumor formation was monitored every 3 days and upon tumor onset, the mice injected with PC3 cells were fed with doxycycline $(0.2 \mathrm{~g} / \mathrm{L})$ water supplemented with $5 \%$ sucrose until the end of the experiment. Necropsies were performed on the animals, and all tissues were examined regardless of their pathological status. Normal and tumor tissue samples were fixed in $10 \%$ neutral-buffered formalin (MilliporeSigma, catalog HT501128) overnight. Then, samples were processed by ethanol dehydration and embedded in paraffin according to standard protocols. Sections $(5 \mu \mathrm{m})$ were prepared for antibody detection and hematoxylin and eosin staining.

\section{Author contributions}

A. Alimonti and A. Alajati developed the concept. A. Alimonti, A. Alajati, and MD designed the experiments, interpreted the data, and wrote the manuscript. A. Alimonti, MD, JC, AR, and IG performed experiments. EP performed the mice experiments. LP and $\mathrm{A}$. Calcinetto read and edited the manuscript. SM, ML, and $\mathrm{RD}$ performed immunohistochemistry and immunofluorescence staining. LC performed fly-related experiments and SH supervised and interpreted the data. GC and WY performed correlation analysis. AS, IF, DNR, JW, VG, and TV performed and analyzed the TMA from prostate cancer patients, and LB and JDB supervised the experiments and interpreted the data. GP, MM, and EC did the preparation and characterization of immunoliposomes. MT and MB performed bioinformatics analysis. VT, GC, and A. Carracedo contributed to the data set analysis. LG contributed to the pathological analysis of mouse prostate tissues. JHR, PW, and HM contributed to pathological analysis of the human TMA used in Figure
1. JPT provided the LNCaP-ABL and Lapc4 human prostate cancer cell lines and made suggestions for experimental interpretation.

\section{Acknowledgments}

This study was supported by European Research Council Consolidator grant 683136; Swiss Cancer League grant KFS4267-082017; the Dr. Josef Steiner Foundation; Swiss Card-Onco-Grant of the Alfred and Annemarie von Sick grant; the Helmut Horten Foundation, SNSF (310030_176045), Prostate Cancer United Kingdom (RIA15-ST2-018), and Fondazione Italiana per la ricerca sul Cancro (AIRC) Investigator Grant (22030) to A. Alimonti's lab. GP and EC were supported by AIRC (IG2017, code 20224). The work of A Carracedo is supported by the Basque Department of Education (IKERTALDE IT1106-16), the Department of Industry, Tourism, and Trade of the Government of the Autonomous Community of the Basque Country, and the Severo Ochoa Excellence Accreditation from Ministero de Ciencia, Innovación y Universidades (MCIU) (SEV-2016-0644). Support was also provided by the MCIU (SAF2016-79381-R, FEDER/EU); the Excellence Networks (SAF2016-81975-REDT); Severo Ochoa Excellence Accreditation (SEV-2016-0644), European Training Networks Project (H2020-MSCA-ITN-308 2016 721532), the Spanish Association Against Cancer (AECC) (IDEAS175CARR; GCTRA18006CARR), La Caixa Foundation (HR17-00094), and the European Research Council (starting grant 336343, proof of concept 754627, consolidator grant 819242).

Address correspondence to: Andrea Alimonti, Institute of Oncology Research, Via V. Vela 6, Bellinzona, Switzerland. Phone: 41.91.8210080; Email: andrea.alimonti@ior.usi.ch.
1. Torre LA, Bray F, Siegel RL, Ferlay J, LortetTieulent J, Jemal A. Global cancer statistics, 2012. CA Cancer J Clin. 2015;65(2):87-108.

2. Watson PA, Arora VK, Sawyers CL. Emerging mechanisms of resistance to androgen receptor inhibitors in prostate cancer. Nat Rev Cancer. 2015;15(12):701-711.

3. Chandrasekar T, Yang JC, Gao AC, Evans CP. Mechanisms of resistance in castration-resistant prostate cancer (CRPC). Transl Androl Urol. 2015;4(3):365-380.

4. Huang Y, Jiang X, Liang X, Jiang G. Molecular and cellular mechanisms of castration resistant prostate cancer. Oncol Lett. 2018;15(5):6063-6076.

5. Taylor BS, et al. Integrative genomic profiling of human prostate cancer. Cancer Cell. 2010;18(1):11-22.

6. Wadosky KM, Koochekpour S. Molecular mechanisms underlying resistance to androgen deprivation therapy in prostate cancer. Oncotarget. 2016;7(39):64447-64470.

7. Jamaspishvili $\mathrm{T}$, et al. Clinical implications of PTEN loss in prostate cancer. Nat Rev Urol. 2018;15(4):222-234.

8. Mulholland DJ, et al. Pten loss and RAS/MAPK activation cooperate to promote EMT and metastasis initiated from prostate cancer stem/progenitor cells. Cancer Res. 2012;72(7):1878-1889.

9. Cho NY, Choi M, Kim BH, Cho YM, Moon KC, Kang GH. BRAF and KRAS mutations in prostatic adenocarcinoma. Int J Cancer. 2006;119(8):1858-1862.

10. Köllermann J, et al. Activating BRAF gene mutations are uncommon in hormone refractory prostate cancer in Caucasian patients. Oncol Lett. 2010;1(4):729-732.

11. Alimonti A, et al. A novel type of cellular senescence that can be enhanced in mouse models and human tumor xenografts to suppress prostate tumorigenesis. J Clin Invest. 2010;120(3):681-693.

12. Chen Z, et al. Crucial role of p53-dependent cellular senescence in suppression of Pten-deficient tumorigenesis. Nature. 2005;436(7051):725-730.

13. Ding Z, et al. SMAD4-dependent barrier constrains prostate cancer growth and metastatic progression. Nature. 2011;470(7333):269-273.

14. Qin J, et al. COUP-TFII inhibits TGF- $\beta$-induced growth barrier to promote prostate tumorigenesis. Nature. 2013;493(7431):236-240.

15. Hooper JD, et al. Subtractive immunization using highly metastatic human tumor cells identifies SIMA135/CDCP1, a 135 kDa cell surface phosphorylated glycoprotein antigen. Oncogene. 2003;22(12):1783-1794.

16. Brown TA, et al. Adhesion or plasmin regulates tyrosine phosphorylation of a novel membrane glycoprotein p80/gp140/CUB domaincontaining protein 1 in epithelia. J Biol Chem. 2004;279(15):14772-14783.
17. Takeda H, Fujimori Y, Kai S, Ogawa H, Nakano T. CD318/CUB-domain-containing protein 1 expression on cord blood hematopoietic progenitors. Exp Ther Med. 2010;1(3):497-501.

18. Bhatt AS, Erdjument-Bromage H, Tempst P, Craik CS, Moasser MM. Adhesion signaling by a novel mitotic substrate of src kinases. Oncogene. 2005;24(34):5333-5343.

19. He Y, Harrington BS, Hooper JD. New crossroads for potential therapeutic intervention in cancer intersections between CDCP1, EGFR family members and downstream signaling pathways. Oncoscience. 2016;3(1):5-8.

20. Uekita T, Sakai R. Roles of CUB domain-containing protein 1 signaling in cancer invasion and metastasis. Cancer Sci. 2011;102(11):1943-1948.

21. Wortmann A, He Y, Deryugina EI, Quigley JP, Hooper JD. The cell surface glycoprotein CDCP1 in cancer--insights, opportunities, and challenges. IUBMB Life. 2009;61(7):723-730.

22. Martin GS. The hunting of the Src. Nat Rev Mol Cell Biol. 2001;2(6):467-475.

23. Pollan SG, et al. Regulation of inside-out $\beta 1$-integrin activation by CDCP1. Oncogene. 2018;37(21):2817-2836.

24. Spassov DS, et al. Phosphorylation of Trask by Src kinases inhibits integrin clustering and functions in exclusion with focal adhesion signaling. $\mathrm{Mol}$ Cell Biol. 2011;31(4):766-782.

25. Spassov DS, Wong CH, Wong SY, Reiter JF, 
Moasser MM. Trask loss enhances tumorigenic growth by liberating integrin signaling and growth factor receptor cross-talk in unanchored cells. Cancer Res. 2013;73(3):1168-1179.

26. Nakashima K, et al. Novel small molecule inhibiting CDCP1-PKC $\delta$ pathway reduces tumor metastasis and proliferation. Cancer Sci. 2017;108(5):1049-1057.

27. Kollmorgen G, et al. Antibody mediated CDCP1 degradation as mode of action for cancer targeted therapy. Mol Oncol. 2013;7(6):1142-1151.

28. Siva AC, et al. Targeting CUB domain-containing protein 1 with a monoclonal antibody inhibits metastasis in a prostate cancer model. Cancer Res. 2008;68(10):3759-3766.

29. Liu F, Yang X, Geng M, Huang M. Targeting ERK, an Achilles' Heel of the MAPK pathway, in cancer therapy. Acta Pharm Sin B. 2018;8(4):552-562.

30. Cima I, et al. Cancer genetics-guided discovery of serum biomarker signatures for diagnosis and prognosis of prostate cancer. Proc Natl Acad Sci USA. 2011;108(8):3342-3347.

31. Fankhauser $\mathrm{CD}$, et al. Comprehensive immunohistochemical analysis of PD-L1 shows scarce expression in castration-resistant prostate cancer. Oncotarget. 2018;9(12):10284-10293.

32. Zellweger T, et al. Estrogen receptor $\beta$ expression and androgen receptor phosphorylation correlate with a poor clinical outcome in hormone-naive prostate cancer and are elevated in castration-resistant disease. Endocr Relat Cancer. 2013;20(3):403-413.

33. Beard C, Hochedlinger K, Plath K, Wutz A, Jaenisch R. Efficient method to generate single-copy transgenic mice by site-specific integration in embryonic stem cells. Genesis. 2006;44(1):23-28.

34. Trotman LC, et al. Pten dose dictates cancer progression in the prostate. PLoS Biol. 2003;1(3):E59.

35. Culí J, Martín-Blanco E, Modolell J. The EGF receptor and $\mathrm{N}$ signalling pathways act antagonistically in Drosophila mesothorax bristle patterning. Development. 2001;128(2):299-308.

36. Khare S, et al. A KCNC3 mutation causes a neu- rodevelopmental, non-progressive SCA13 subtype associated with dominant negative effects and aberrant EGFR trafficking. PLOS ONE. 2017;12(5):e0173565.

37. Brumby AM, Richardson HE. Using Drosophila melanogaster to map human cancer pathways. Nat Rev Cancer. 2005;5(8):626-639.

38. Bülow MH, Bülow TR, Hoch M, Pankratz MJ, Jünger MA. Src tyrosine kinase signaling antagonizes nuclear localization of FOXO and inhibits its transcription factor activity. Sci Rep. 2014;4:4048.

39. Dodson GS, Guarnieri DJ, Simon MA. Src64 is required for ovarian ring canal morphogenesis during Drosophila oogenesis. Development. 1998;125(15):2883-2892.

40. Alimonti A, et al. Subtle variations in Pten dose determine cancer susceptibility. Nat Genet. 2010;42(5):454-458.

41. Furstoss O, Dorey K, Simon V, Barilà D, SupertiFurga G, Roche S. c-Abl is an effector of Src for growth factor-induced c-myc expression and DNA synthesis. EMBO J. 2002;21(4):514-524.

42. Jain S, et al. Src inhibition blocks c-Myc translation and glucose metabolism to prevent the development of breast cancer. Cancer Res. 2015;75(22):4863-4875.

43. Barone MV, Courtneidge SA. Myc but not Fos rescue of PDGF signalling block caused by kinase-inactive Src. Nature. 1995;378(6556):509-512.

44. Lunardi A, et al. A co-clinical approach identifies mechanisms and potential therapies for androgen deprivation resistance in prostate cancer. Nat Genet. 2013;45(7):747-755.

45. Di Mitri D, et al. Tumour-infiltrating Gr-1+ myeloid cells antagonize senescence in cancer. Nature. 2014;515(7525):134-137.

46. Hennequin LF, et al. N-(5-chloro-1,3-benzodioxol-4-yl)-7-[2-(4-methylpiperazin-1-yl)ethoxy]-5(tetrahydro-2H-pyran-4-yloxy)quinazolin-4amine, a novel, highly selective, orally available, dual-specific c-Src/Abl kinase inhibitor. JMed
Chem. 2006;49(22):6465-6488.

47. Carver BS, et al. Reciprocal feedback regulation of PI3K and androgen receptor signaling in PTEN-deficient prostate cancer. Cancer Cell. 2011;19(5):575-586.

48. Burton DG, et al. Androgen deprivation-induced senescence promotes outgrowth of androgenrefractory prostate cancer cells. PLOS ONE. 2013;8(6):e68003.

49. Campisi J. Aging and cancer: the double-edged sword of replicative senescence. J Am Geriatr Soc. 1997;45(4):482-488.

50. Jain RK. Normalization of tumor vasculature: an emerging concept in antiangiogenic therapy. Science. 2005;307(5706):58-62.

51. Maeda H, Nakamura H, Fang J. The EPR effect for macromolecular drug delivery to solid tumors: Improvement of tumor uptake, lowering of systemic toxicity, and distinct tumor imaging in vivo. Adv Drug Deliv Rev. 2013;65(1):71-79.

52. Maeda H, Tsukigawa K, Fang J. A retrospective 30 years after discovery of the enhanced permeability and retention effect of solid tumors: next-generation chemotherapeutics and photodynamic therapy--problems, solutions, and prospects. Microcirculation. 2016;23(3):173-182.

53. Yang L, et al. Dysregulated expression of cell surface glycoprotein $\mathrm{CDCP} 1$ in prostate cancer. Oncotarget. 2015;6(41):43743-43758.

54. Chandran UR, et al. Gene expression profiles of prostate cancer reveal involvement of multiple molecular pathways in the metastatic process. BMC Cancer. 2007;7:64.

55. Varambally S, et al. Integrative genomic and proteomic analysis of prostate cancer reveals signatures of metastatic progression. Cancer Cell. 2005;8(5):393-406.

56. Grasso CS, et al. The mutational landscape of lethal castration-resistant prostate cancer. Nature. 2012;487(7406):239-243.

57 . Lefort K, et al. Dual tumor suppressing and promoting function of Notch1 signaling in human prostate cancer. Oncotarget. 2016;7(30):48011-48026. 